\title{
Coherence Spectroscopy Investigations of the Low-Frequency Vibrations of Heme: Effects of Protein-Specific Perturbations
}

\author{
Flaviu Gruia ${ }^{\dagger}$, Minoru Kubo ${ }^{\dagger}$, Xiong $\mathrm{Ye}_{\star}^{\dagger}$, Dan lonascu ${ }^{\dagger}$, Changyuan Lu†, Robert K. Poole $\$$, \\ Syun-Ru Yeh $\mp$, and Paul M. Champion ${ }^{*}, \dagger$ \\ tDepartment of Physics and Center for Interdisciplinary Research on Complex Systems, \\ Northeastern University, Boston, Massachusetts 02115 \\ ‡Department of Physiology and Biophysics, Albert Einstein College of Medicine, Bronx, New York \\ 10461 \\ §Department of Molecular Biology and Biotechnology, University of Sheffield, Sheffield S10 2TN, \\ United Kingdom
}

\begin{abstract}
Femtosecond coherence spectroscopy is used to probe the low-frequency $\left(20-200 \mathrm{~cm}^{-1}\right)$ vibrational modes of heme proteins in solution. Horseradish peroxidase (HRP), myoglobin (Mb), and Campylobacter jejuni globin (Cgb) are compared and significant differences in the coherence spectra are revealed. It is concluded that hydrogen bonding and ligand charge do not strongly affect the lowfrequency coherence spectra and that protein-specific deformations of the heme group lower its symmetry and control the relative spectral intensities. Such deformations potentially provide a means for proteins to tune heme reaction coordinates, so that they can perform a broad array of specific functions. Native HRP displays complex spectral behavior above $\sim 50 \mathrm{~cm}^{-1}$ and very weak activity below $\sim 50 \mathrm{~cm}^{-1}$. Binding of the substrate analog, benzhydroxamic acid, leads to distinct changes in the coherence and Raman spectra of HRP that are consistent with the stabilization of a heme water ligand. The $\mathrm{CN}$ derivatives of the three proteins are studied to make comparisons under conditions of uniform heme coordination and spin-state. $\mathrm{MbCN}$ is dominated by a doming mode near 40 $\mathrm{cm}^{-1}$, while HRPCN displays a strong oscillation at higher frequency $\left(96 \mathrm{~cm}^{-1}\right)$ that can be correlated with the saddling distortion observed in the X-ray structure. In contrast, $\mathrm{CgbCN}$ displays lowfrequency coherence spectra that contain strong modes near 30 and $80 \mathrm{~cm}^{-1}$, probably associated with a combination of heme doming and ruffling. HRPNO displays a strong doming mode near 40 $\mathrm{cm}^{-1}$ that is activated by photolysis. The damping of the coherent motions is significantly reduced when the heme is shielded from solvent fluctuations by the protein material and reduced still further when $\mathrm{T} \lesssim 50 \mathrm{~K}$, as pure dephasing processes due to the protein-solvent phonon bath are frozen out.
\end{abstract}

\section{Introduction}

Heme proteins are involved in a wide range of biochemical processes including transport and storage of small molecules, sensing, signaling, redox reactions, catalysis, and control of gene expression. ${ }^{1-8}$ These proteins have a heme prosthetic group (iron protoporphyrin IX, FePPIX) at the active site, which establishes at least one covalent bond to the surrounding protein matrix through a proximal axial ligand to the central iron atom. In some cases, both axial positions are occupied by amino acid ligands (e.g., cytochrome c, ${ }^{7,9} \mathrm{CooA},{ }^{10} \mathrm{CBS}^{11}$ ), while covalent 
linkages to the heme periphery also occur in the cytochrome family. ${ }^{7,9}$ Depending on the specific system, the central iron atom can bind various functionally important exogenous ligands, such as molecular oxygen, nitric oxide, carbon monoxide, or water. The coordination and the oxidation state of the central iron atom vary between different types of heme proteins and can change during the catalytic cycle. However, given the enormous range of biochemical processes mediated by heme proteins, there are surprisingly small variations in the chemical composition of the heme group. Although it has been established that the amino acid axial ligands play a major role in the functionality of heme proteins, there are many heme proteins that share both the same heme and the same axial ligands yet display significantly different functions. The fact that a specific function can be strongly altered by mutations in the amino acid sequence and tertiary structure of the surrounding protein ${ }^{12-14}$ suggests that the protein matrix is able to tune and modulate heme functionality. For example, it has been suggested ${ }^{15-18}$ that the interaction between the adjacent protein material and the normally planar heme group can lead to out-of-plane structural distortions that may be of functional importance.

In this work we use femtosecond coherence spectroscopy (FCS) to document low-frequency modes in horseradish peroxidase (HRP), myoglobin (Mb), and Campylobacter jejuni globin $(\mathrm{Cgb})$ that reflect heme geometric distortions by the protein architecture. FCS is a "pumpprobe" technique that employs the large bandwidth associated with ultrafast laser pulses to monitor vibrational coherences induced within the sample of interest. The theoretical details of this time domain spectroscopic technique can be found elsewhere. ${ }^{19-27}$ One important advantage of the FCS technique over more traditional frequency domain vibrational spectroscopies is its ability to monitor very low-frequency vibrational modes $\left(20-200 \mathrm{~cm}^{-1}\right)$ that are otherwise inaccessible to experimental detection in the aqueous environment. Vibrational modes in the region below $200 \mathrm{~cm}^{-1}$ are easily activated by thermal fluctuations $\left(k_{\mathrm{B}} T \sim 200 \mathrm{~cm}^{-1}\right.$ at room temperature), and the functionally important heme vibrations are likely to lie within this range. For example, it has recently been demonstrated how the hemedoming motion near $40 \mathrm{~cm}^{-1}$ influences diatomic ligand binding. ${ }^{28}$

To assess the importance of the protein environment on the low-frequency vibrational spectrum of the heme, we carried out a series of experiments on Mb, HRP (isoenzyme C), and Cgb. Each of these heme proteins has a b-type heme in the active site that is covalently bound to the rest of the protein through a histidine amino acid (His 93 in Mb, His170 in HRP, and His85 in Cgb). $\mathrm{Mb}$ and $\mathrm{HRP}$ have significantly different roles: $\mathrm{Mb}$ is a member of the globin family and is mostly involved in oxygen storage in the muscle cells of vertebrates, while HRP is a peroxidasetype heme protein that has important catalytic functions in plants. In the native state of $\mathrm{Mb}$, the central iron atom is reduced $\left(\mathrm{Fe}^{2+}\right)$ and can be high-spin, five-coordinated (deoxyMb) or low-spin, six-coordinated ( $\mathrm{MbL} ; \mathrm{L}=\mathrm{CO}, \mathrm{O}_{2}, \mathrm{NO}$ ). In the native state, $\mathrm{HRP}$ is thought to have a five- and six-coordinate iron, in a high- or mixed-spin state. ${ }^{29-32}$ The resting state ferric heme iron in HRP explores higher oxidation states $(+4)$ during the catalytic cycle. Cgb is a singledomain hemoglobin from the bacterium Campylobacter jejuni that has been proposed to protect the bacterium against $\mathrm{NO}$ attack. ${ }^{33}$

In the experiments described below, we attempted to match the coordination, oxidation, and spin states of the protein samples considered for comparison. For example, we used the ferricyanide adducts to eliminate ambiguities associated with the coordination and spin states of the different samples. Even under these conditions, we found that these apparently similar heme proteins show very different coherence spectra. Most notably, the HRP species shows very little activity below $50 \mathrm{~cm}^{-1}$, whereas the $\mathrm{Mb}$ samples display strong low-frequency modes in this region. Given the identical spin, oxidation, and axial ligation of the ferricyanide complexes, we suggest that distortion of the heme geometry, arising from its local interactions with the protein matrix, leads to dramatic changes in the low-frequency region of the vibrational 
spectrum. This study demonstrates that FCS is a sensitive probe of the out-of-plane geometric distortions that are induced when the heme is inserted into different protein environments. These distortions can be quantified by using normal coordinate structural decomposition (NSD). ${ }^{34}$ The effect of the distortions is to lower the inherent symmetry of the heme and activate out-of-plane low-frequency modes that fall in the region $\leqslant k_{\mathrm{B}} T$. These modes are likely to play a role in heme protein function, either by helping to "trap" product states once they are formed $^{35}$ or as direct reaction coordinates. ${ }^{28}$

\section{Experimental Section}

\section{Sample Preparation}

Horse heart myoglobin $(\mathrm{Mb})$, horseradish peroxidase isoenzyme $\mathrm{C}(\mathrm{HRP})$, benzhydroxamic acid (BHA), and potassium cyanide (KCN) were purchased from Sigma Chemical Co. (St. Louis, $\mathrm{MO}$ ) and used directly without further purification. The ferric heme protein samples were prepared by dissolving the lyophilized protein powder in potassium phosphate buffer $(\mathrm{pH}$ $7.0,0.1 \mathrm{M})$ and adjusting the sample concentration to the required final optical density $(\sim 1$ O.D./mm of path length) by dilution.

The ferric HRP/BHA complex was obtained by adding small aliquots of concentrated BHA solution to the ferric HRP sample. Given the high affinity of BHA for HRP, the final BHA concentration was approximately $100 \mu \mathrm{M}$. The HRP-CN and $\mathrm{Mb}-\mathrm{CN}$ adducts were prepared by adding small aliquots of $1 \mathrm{M} \mathrm{KCN}$ solution to the ferric protein samples. The final concentration of $\mathrm{CN}$ was approximately $300 \mu \mathrm{M}$ (slight excess). The ferric HRP/BHA-NO sample was formed by preparing the HRP/BHA complex, as previously described, and then bubbling $\mathrm{NO}$ gas through it for approximately 1 min under continuous agitation.

The Campylobacter globin $(\mathrm{Cgb})$ sample from the microaerophilic bacterium Campylobacter jejuni was obtained as discussed previously. ${ }^{36}$ The ferric $\mathrm{Cgb}$ sample was dissolved in Tris buffer $(50 \mathrm{mM}, \mathrm{pH} 7.4)$ to a final heme concentration of approximately $75 \mu \mathrm{M}$. The cyanide adduct was obtained by adding small aliquots of $1 \mathrm{M} \mathrm{KCN}$ solution to the ferric protein samples.

To prepare the reduced species of $\mathrm{Mb}, \mathrm{HRP}$ and $\mathrm{Cgb}$, the proteins were first dissolved in the appropriate buffer (potassium phosphate buffer $\mathrm{pH} 7.0,0.1 \mathrm{M}$ for $\mathrm{Mb}$ and HRP, Tris buffer $\mathrm{pH} 7.4,50 \mathrm{mM}$ for $\mathrm{Cgb}$ ) and degassed for $20 \mathrm{~min}$ under an argon atmosphere. The concentration of protein was adjusted so that the final absorbance of the reduced species was about 1 O.D./ $\mathrm{mm}$ of path length at the pump wavelength. Subsequently, the samples were transferred into a glovebox and $3 \mu \mathrm{L}$ of $1 \mathrm{M}$ sodium dithionate $\left(\mathrm{Na}_{2} \mathrm{O}_{4} \mathrm{~S}_{2}\right)$ was added to $250 \mu \mathrm{L}$ of buffered sample to obtain the deoxy species.

To obtain the $\mathrm{NO}$ adducts, $2 \mu \mathrm{L}$ of $1 \mathrm{M}$ sodium nitrate $\left(\mathrm{Na}_{2} \mathrm{NO}_{2}\right)$ solution was added to the deoxy sample. The reduced samples were then transferred into the sample cell and carefully insulated against oxygen contamination. For low temperature measurements a mixture of glycerol (75\%) and buffer (25\%) was used for sample dilution. The final concentration was adjusted so the optical density of the sample at the excitation wavelength was approximately 1 O.D. in a $1 \mathrm{~mm}$ path length cell.

The absorption spectra of all samples were recorded (using a Hitachi U-3410 spectrophotometer) after the preparation procedure to confirm that all chemical modifications were achieved and that they were complete. Absorption spectra were also taken after the experiments were concluded to check for possible photochemical damage and none was observed. 


\section{Laser System}

The laser system used for the FCS measurements consisted of a tunable (750-960nm) Tisapphire oscillator (MIRA 900, Coherent Inc., Santa Clara, CA) pumped by a diode laser (Verdi 10, Coherent, Inc., Santa Clara, CA). The oscillator was able to generate 50-100 fs pulses at a repetition rate of $76 \mathrm{MHz}$, with energy of $\sim 10 \mathrm{~nJ} /$ pulse. To resonantly excite the samples in the Soret band, the IR output of the laser was frequency doubled in a $250 \mu \mathrm{m} \beta$-barium borate (BBO) crystal and then chirp compensated by a pair of SF10 prisms to within $10 \%$ of the transform time-bandwidth limit. Subsequently, the laser light was split into a pump arm and a probe arm, with a power ratio of 2:1 between the two. The pump beam was modulated using an accustooptic modulator (AOM; Neos Technologies (Melbourne, FA)) at 1.5 MHz. Before reaching the sample, the pump and probe beam polarizations were adjusted to be perpendicular. Both beams were focused into the spinning sample cell using a three inch achromatic lens in a near parallel geometry. The time delay between the pump and probe-pulses was controlled by a Newport Klinger (Irvine, CA) translation stage in steps of $1 \mu \mathrm{m}$ (6.66 fs steps in the time domain). After the sample, the beams were recollimated and the pump light was spatially blocked (using a pinhole that allows only the probe beam to pass) and extinguished by a polarization analyzer that was oriented accordingly.

The detection step of the experimental procedure allows us to focus on different frequency ranges of the coherent signal. In an "open band" detection scheme, we employ a Si photodiode to measure the entire spectral bandwidth of the probe-pulse. This kind of measurement integrates over all frequencies and results in better fidelity for the detection of low-frequency modes. ${ }^{21,37}$ The "detuned" or "dispersed" detection scheme, on the other hand, leads to stronger relative enhancement of the higher frequency regions of the coherent signal. ${ }^{21,37-39}$ In this latter case, a photomultiplier tube (PMT) coupled to a monochromator was employed for detection, resulting in increased amplitude and superior detection of the higher frequency components of the third order polarization signal. This occurs because the induced polarization oscillates at the optical carrier frequency \pm the coherent vibrational frequencies. Thus, as the detection is moved away from the optical carrier frequency, higher frequency modes are selectively detected. When the detuning conditions are chosen so they selectively augment modes above $200 \mathrm{~cm}^{-1}$, we can compare the FCS results directly with the frequency domain Raman spectra. The excited electronic state coherence signals do not contribute to the heme coherence spectra because such states are very short-lived ( $<100 \mathrm{fs})$. On the other hand, "product" states, which correspond to ground electronic product states of a photochemical reaction, such as $\mathrm{Mb}$ in $\mathrm{MbL} \rightarrow \mathrm{Mb}+\mathrm{L}$, will contribute, and signals from such samples will be a superposition of the reactant and product state coherences. The various contributions to the observed signal will depend on the quantum yield for the reaction and the probe laser wavelength resonance conditions.

The output of the detector under either open band or detuned conditions (i.e., using either the photodiode or the photomultiplier) was passed through a lock-in amplifier (LIA) (Stanford SR844) that retrieves and amplifies the signal associated with the pump modulation frequency (1.5 MHz, set using the LIA output as a clock). Given this processing step, it is imperative to take precautions so that the pump light is extinguished, using spatial and polarization filtering, before reaching the detector. The pump light that leaks into the detector appears as a constant offset in the detected signal, which can adversely affect the dynamic range of the LIA.

\section{Low Temperature Measurements}

To carry out temperature dependence studies, we developed a novel scanning method that removes the technical limitations related to refreshing the sample in a cryostat. Sample refresh is needed in order to avoid photo-damage, thermal lensing, and incomplete reset to equilibrium between pulse pairs. The details of this technique are presented elsewhere. ${ }^{40}$ Normally, we use 
a spinning sample cell for sample refresh, but for the low temperature measurements, spinning the sample in the cryostat is very difficult. Instead we use an off-axis focusing lens that spins in front of the sample and moves the laser beams in a circular path over the frozen sample without the need of moving the sample itself. There is additional noise introduced by this scanning method and also by optical imperfections associated with the frozen sample, which decrease the signal/noise $(\mathrm{S} / \mathrm{N})$ ratio and tend to wash out the higher frequencies to some degree. ${ }^{40}$ Nevertheless, the benefits of being able to efficiently probe samples at cryogenic temperatures surpass the negative side effects on the $\mathrm{S} / \mathrm{N}$ ratio.

\section{Data Analysis}

The experimental FCS data have two components that result from different mechanisms, population transfer and vibrational coherence. The dominant population transfer components usually have a monotonic decay behavior. Although these components carry important information regarding various dynamic processes (e.g., ligand rebinding dynamics, cooling and/or spectral diffusion of the line shape) they must be removed in order to reveal the residual coherence signal. Due to the large amplitude of the electronic population signals (generally about 2 orders of magnitude larger than the vibrational coherence signal), special precautions have to be taken during both the digitization/recording of the signal and the fitting procedure. The digitization of the experimental signal was done by the LIA on a 24 bit scale so that it allowed for a sufficient dynamic range even for low amplitude coherence signals. The data analysis followed a sequential approach. We first employed a maximum entropy method (MEM) algorithm to retrieve the oscillatory signal because MEM removes assumptions regarding the number of exponential decay processes that might be present. The oscillatory coherence signal comes from the superposition of vibrational states coupled to the resonant electronic transition. This coherent superposition is lost after a few picoseconds following the excitation due to both homogeneous and inhomogeneous decoherence processes that cause the oscillatory signal to damp as a function of time. To quantify the coherent signals, the oscillatory signal left after the removal of the monotonic decay terms was fit to a sum of damped cosine functions,

$$
I(t)=\sum_{i} A_{i} e^{-\gamma_{i} t} \cos \left(\omega_{i} t+\varphi_{i}\right)
$$

using a linear predictive single value decomposition (LPSVD) algorithm. Given the fact that a small amount of noise is always present in the experimental data, we use the low frequencies observed in the Raman spectrum as a control that helps us avoid over- or under-fitting the data.

We first analyze the detuned data (which optimizes the detection of the higher frequencies within the pulse bandwidth) to make sure that the global fitting parameters (such as the number of oscillators and time domain of the data) are properly correlated with the frequencies determined independently from the Raman spectrum. The power spectra obtained from the detuned measurements generally extend below $200 \mathrm{~cm}^{-1}$, which represents the practical lower limit cutoff for heme protein resonance Raman measurements. After analysis of the detuned data, we consider the open band experiment and compare the frequencies in the overlapping regions. The analysis of open band data starts by selecting a low number of oscillations and then gradually increasing that number until the overall resolved frequencies match the results obtained through detuned measurements and Raman spectroscopy. The relative intensities of the coherences are a strong function of the laser pulse bandwidth and detuning conditions. The LPSVD spectra, as presented, are not corrected for the Gaussian-distributed relative pumping efficiencies within the bandwidth of the laser pulse. The cross-correlation pulse widths are typically kept near $60 \pm 10 \mathrm{fs}$, to selectively enhance the lower frequency modes. Examination of the data from numerous independent samples ${ }^{41}$ has generally produced very good 
reproducibility and strong correlations between the frequencies extracted using the three experimental conditions (Raman, detuned FCS, and open band FCS). The phase $\left(\varphi_{\mathrm{i}}\right)$ cannot be determined from Raman measurements. We estimate that, for mode frequencies below 100 $\mathrm{cm}^{-1}$, the phases determined to be near $(\sim)$ a particular value will have an error at or below $\pm \pi / 6\left( \pm 30^{\circ}\right)$.

\section{Results}

Figure 1 shows the absorption spectra of some of the HRP (panels A and B), Mb (panel C), and $\mathrm{Cgb}$ (panel D) samples considered in this study. In its native ferric form, HRP displays a Soret band with a maximum at $402 \mathrm{~nm}$ and a marked shoulder on the blue side. The interpretation of this spectrum remains somewhat obscure. ${ }^{5,29,42}$ It is likely that the two bands indicate the presence of two different species, involving distal pocket water and a mixture of five- and six-coordinate heme. As can be seen in Figure 1C, a five-coordinate ferric $\mathrm{Mb}$ absorption spectrum, with no distal water, has been measured for the H64L mutant. ${ }^{43,44}$ It displays a broad Soret band that peaks near $390 \mathrm{~nm}$, which is suggestive of the possibility that the shoulder near $390 \mathrm{~nm}$ in native HRP represents the population of the five-coordinate ferric heme, while the peak near $403 \mathrm{~nm}$ reflects the presence of water-bound six-coordinate material. The five/six-coordinate equilibrium is shifted by the addition of benxhydroxamic acid (BHA), a substrate-like inhibitor of HRP, which favors a six-coordinated water-bound species. ${ }^{30}$ When BHA is present (HRP/BHA), the absorption spectrum is more homogeneous, with a Soret peak at $408 \mathrm{~nm}$ (the shift to $403 \mathrm{~nm}$ in the absence of BHA can be simulated by superimposing the five- and six-coordinate lineshapes). The HRP/BHA peak at $408 \mathrm{~nm}$ closely resembles the spectrum of ferric (met) $\mathrm{Mb}$ ( $409 \mathrm{~nm}$ Soret maximum), which has a six-coordinate high-spin $(S=5 / 2)$ iron with a bound water molecule.

The absorption spectra of the six-coordinate ferric cyanide-bound species (HRPCN, MbCN) are nearly identical with Soret peaks at approximately $423 \mathrm{~nm}$, while CgbCN has its Soret peak at $418 \mathrm{~nm}$. The reduced five-coordinate samples of $\mathrm{Mb}, \mathrm{HRP}$, and $\mathrm{Cgb}$ are also very similar, showing Soret peaks at 435, 437, and 432 nm, respectively. Panel B of Figure 1 shows the absorption spectra of the ferric and ferrous nitric oxide (NO)-bound species of HRP, which both have Soret peaks at $422 \mathrm{~nm}$. In panel D, we display the Soret bands of the Cgb sample in the ferric, ferrous and $\mathrm{CN}$ bound states. It is noteworthy that the ferric Cgb sample absorbs near $398 \mathrm{~nm}$, similar to the five-coordinate ferric H64LMb mutant, and that it has been assigned as a primarily five-coordinate heme using Raman spectroscopy. ${ }^{36}$ However, there is a weak structure associated with the Soret line shape of ferric $\mathrm{Cgb}$ that is suggestive of what is seen in ferric HRP, potentially indicating the presence of a small fraction of six-coordinate material.

We carried out an extended series of FCS experiments on ferric Mb and ferric HRP (with and without BHA) using both the open band and the detuned detection techniques. All measurements were performed with identical laser pulses $(418 \mathrm{~nm}, 60 \mathrm{fs})$ to optimize the comparisons between the coherence signals of the three samples. Figure 2 shows a set of comparative Raman, detuned, and open band FCS results for samples of ferric Mb (panel A), ferric HRP (panel B), and ferric HRP/BHA (panel C). The insets display the oscillatory components of the data used to generate the spectra shown in the lower two traces. As expected, the Raman data do not effectively resolve spectral features in the range below $\sim 200 \mathrm{~cm}^{-1}$. This is due to experimental constraints that mainly involve Rayleigh/quasielastic light scattering and Raman notch filter effects (the latter are more pronounced in the HRP Raman spectra). On the other hand, the coherence data demonstrate that there is considerable vibrational activity below $200 \mathrm{~cm}^{-1}$. The lowest frequency oscillations are optimally detected in an open band measurement, while the detuned detection bridges the gap between the open band region and the higher frequency region probed by Raman spectroscopy. As observed in all three panels 
of Figure 2, there is a good correlation between the separate experiments: detuned FCS (blue curves), Raman (red curves), and open band FCS (green curves).

The metMb sample shows particularly impressive agreement between the coherence and the Raman spectra, and for comparison, we have also inserted the Raman spectrum of the fivecoordinate $\mathrm{H} 64 \mathrm{~L} \mathrm{Mb}$ mutant in the $600-800 \mathrm{~cm}^{-1}$ region. The depolarized $v_{15}$ mode (near 750 $\mathrm{cm}^{-1}$ ) of the H64L mutant sample shows a dramatic gain of intensity when the water ligand is lost. A similar effect is seen in panels B and C when the Raman spectra of HRP and HRP/BHA are compared. This demonstrates that the change in the $750 \mathrm{~cm}^{-1}$ mode intensity can be used as a monitor of the conversion between the five-and six-coordinate $\mathrm{H}_{2} \mathrm{O}$-bound heme species. When BHA binds it stabilizes the water molecule and suppresses the $750 \mathrm{~cm}^{-1}$ mode. ${ }^{43,44}$

A direct comparison of the open band coherence spectra of ferric species of $\mathrm{Mb}$ and $\mathrm{HRP}$ with and without BHA is shown in the upper three panels of Figure 3 (measured at $418 \mathrm{~nm}$ ). The frequencies of $\mathrm{Mb}$ and HRP are summarized in Table 1 and Table 2. The differences observed in the coherence spectra of the three samples are significant, and surprisingly, these differences persist even for the two species that have very similar absorption spectra (HRP/BHA and metMb). While the coherence spectrum of metMb has a dominant component at $39 \mathrm{~cm}^{-1}$, the spectrum of HRP/BHA shows its strongest mode at $102 \mathrm{~cm}^{-1}$. The native HRP measured under identical conditions shows a very congested low-frequency landscape, with major components at 94,144 , and $191 \mathrm{~cm}^{-1}$. Given that the native enzyme contains a mixture of five- and sixcoordinated species, spectral crowding is expected for this sample.

To interrogate the five-coordinate species of the native enzyme, which probably absorbs near $390 \mathrm{~nm}^{43}$ (as seen in Figure 1C), we also carried out coherence measurements with $405 \mathrm{~nm}$ excitation (lower panels of Figure 3). The strongest oscillations in the HRP spectrum appear at 77 and $196 \mathrm{~cm}^{-1}$, which is conspicuously different from the data recorded with $418 \mathrm{~nm}$ excitation. The HRP/BHA Soret absorption spectrum appears to be more homogeneous, and the coherence spectrum excited at $418 \mathrm{~nm}$ displays strong modes at 102,170 , and $187 \mathrm{~cm}^{-1}$. Moving the excitation to $405 \mathrm{~nm}$ leads to changes in the relative amplitudes of the HRP/BHA sample, as expected theoretically, ${ }^{21}$ but additional modes also appear, which is not expected for a homogeneous sample. For example, a dominant new oscillation can be seen at $73 \mathrm{~cm}^{-1}$, close to the strong $77 \mathrm{~cm}^{-1}$ mode observed in native HRP measured at $405 \mathrm{~nm}$. The mode at $37 \mathrm{~cm}^{-1}$ in the HRP/BHA sample is intensified at $405 \mathrm{~nm}$ and is slightly shifted with respect to $418 \mathrm{~nm}$ excitation. Generally, these observations suggest that, even in the presence of BHA, the heme in HRP fluctuates between at least two different conformations (or mixed spin states).

The bottom panel of Figure 3 shows the coherence spectrum of the five-coordinate H64L Mb mutant, excited at $405 \mathrm{~nm}$, which is close to the Soret band maximum at $390 \mathrm{~nm}$. There are significant differences between the coherence spectrum of this sample and the native ferric HRP sample excited at $405 \mathrm{~nm}$ (which should selectively probe the five-coordinate population). This, along with the significant spectral differences between the six-coordinate HRP and Mb samples (upper two panels of Figure 3), strongly indicates that the heme coordination state is not responsible for the very different coherence spectra found for HRP and Mb.

The absorption spectra suggest that the native ferric HRP sample is a mixture of five- and sixcoordinate material, with the likelihood of mixed spin-states. ${ }^{45}$ As a result, comparison of native HRP to the six-coordinate high-spin ferric metMb system could be complicated by the presence of multiple species. To help clarify the situation, we decided to probe the lowfrequency vibrational spectrum by comparing hemes with identical spin, oxidation, and axial ligation states. The absorption spectra suggest that both the ferricyanide species (sixcoordinate, low-spin) and the ferrous deoxy species (five-coordinate, high-spin) are excellent 
targets for protein-specific comparative studies where the heme is fixed in a well-defined coordination and spin-state.

Figure 4A shows the result of FCS experiments carried out under similar conditions using the ferricyanide derivatives of $\mathrm{HRP}, \mathrm{Cgb}$, and $\mathrm{Mb}$. The frequencies of these species are presented in Table 1-Table 3. All samples have very good stability and display strong coherence signals. The analysis of the experimental results shows that, while the MbCN sample has a dominant mode at $40 \mathrm{~cm}^{-1}$, the HRPCN sample shows very limited activity below $\sim 70 \mathrm{~cm}^{-1}$ and the main component of the coherence spectrum appears at $96 \mathrm{~cm}^{-1}$. As reported elsewhere, ${ }^{41}$ the phase of the $40 \mathrm{~cm}^{-1}$ mode of $\mathrm{MbCN}$, as given in eq 1, is anomalous and displays a sharp transition from $+\pi / 2$ to $-\pi / 2$ near $418 \mathrm{~nm}$. This indicates that the initial pump-induced nonequilibrium coherent wavepacket is dominated by momentum transfer rather than the coordinate displacement normally observed for resonant excitation. ${ }^{21}$ In contrast, the HRP mode at $96 \mathrm{~cm}^{-1}$ has a roughly constant phase $(\varphi \sim 0)$, which is indicative of a coordinate displacement and a field-driven ground-state coherence. The $\mathrm{CgbCN}$ sample displays a coherence spectrum that is qualitatively intermediate between HRPCN and MbCN. There are two dominant modes, one at $30 \mathrm{~cm}^{-1}$, with a phase near zero, and another at $79 \mathrm{~cm}^{-1}$, with a phase near $\pi / 6$. As can be seen in Figure 4B, the FCS frequencies detected above $200 \mathrm{~cm}^{-1}$ in HRPCN correlate very well with the Raman data, with the unexplained exception of the modes near $265-280 \mathrm{~cm}^{-1}$.

Figure 5 presents the results of the open band coherence measurements carried out on the fivecoordinate ferrous forms of HRP, $\mathrm{Cgb}$, and $\mathrm{Mb}$. To account for the slight difference in the absorption spectra of the samples (437 vs $432 \mathrm{vs} 435 \mathrm{~nm}$ ), we performed the experiments with 432,425 , and $430 \mathrm{~nm}$ excitation, respectively. Even though the spin, oxidation, and coordination states of the samples are identical, the differences in their low-frequency spectra continue the same trend as observed previously for the ferric species. Ferrous Mb excited at $430 \mathrm{~nm}$ shows a strong mode near $46 \mathrm{~cm}^{-1}$ along with distinctive features at 98, 119, and 219 $\mathrm{cm}^{-1}$. Ferrous HRP, on the other hand, has weak activity in the region below $\sim 50 \mathrm{~cm}^{-1}$, with the main vibrations tightly packed at $57,78,104$, and $128 \mathrm{~cm}^{-1}$. Like $\mathrm{Mb}$, the ferrous $\mathrm{Cgb}$ sample has strong activity below $\sim 50 \mathrm{~cm}^{-1}$ (the strongest modes appear at 31 and $54 \mathrm{~cm}^{-1}$, with phases near zero and $\pi / 2$, respectively), and it also displays important features at higher frequencies, namely, 87,119 , and $142 \mathrm{~cm}^{-1}$. We are able to clearly identify the Fe-His stretching modes $36,42,46$ for all three proteins at $219 \mathrm{~cm}^{-1}$ (Fe-His93 in Mb), $253 \mathrm{~cm}^{-1}(\mathrm{Fe}-$ His 85 in $\mathrm{Cgb}$ ), and $248 \mathrm{~cm}^{-1}$ (Fe-His170 in HRP). The slightly higher frequencies of the FeHis mode in HRP and $\mathrm{Cgb}$, compared to $\mathrm{Mb}$, are due to the proximal hydrogen bonds formed between Asp247/His 170 in $\mathrm{HRP}^{47}$ and between Tyr84/Glu134/His85 in Cgb. ${ }^{36}$ The possibility that these $\mathrm{H}$-bonds are responsible for the differences in low-frequency activity of the three proteins is examined further below.

Both ferric and ferrous HRP are able to bind NO as an axial ligand, which is easily photolyzed. 48 If a significant amount of slow bimolecular rebinding is present in the sample, the photolysis process can negatively affect the FCS experiment. Because of the $76 \mathrm{MHz}$ repetition rate of the laser system, a slow rebinding process can result in the appearance of a mixture of bound and unbound species. While ferrous HRP displays only a very fast NO geminate rebinding time scale, ${ }^{48}$ the ferric HRP-NO has a slower bimolecular component that makes FCS experiments difficult. We bypass this difficulty by using the BHA-bound sample, which has the same absorption spectrum for the NO adduct as the sample without BHA. HRP/BHA has no bimolecular phase because the BHA molecule blocks the access between the heme pocket and the surrounding solvent. ${ }^{48}$ Figure $6 \mathrm{~A}$ shows the coherence spectra of ferrous samples of HRP and HRPNO. The measurements were performed with identical pulses (432 nm, $60 \mathrm{fs}$ ) to facilitate comparison of the data. There is generally a one-to-one correspondence (within $\pm 5 \mathrm{~cm}^{-1}$ ) between the oscillation frequencies seen in the two samples. However, there is one 
exception. A strong mode near $40 \mathrm{~cm}^{-1}$ appears to be "turned on" by the photolysis process. This same effect is also seen in the ferric HRP/BHA-NO sample, with an even more dramatic change in amplitude. As can be seen in Figure 6B, the coherence spectrum of ferric HRP/BHANO sample is dominated by an oscillation that appears at $42-44 \mathrm{~cm}^{-1}$.

In Figure 6, both the ferrous and ferric NO ligated species are compared with the corresponding five-coordinated species, which is the expected product state. The ferrous HRP product is a homogeneous sample that reveals the spectrum of the five-coordinated material over the whole range of excitation wave-lengths. On the other hand, the BHA-bound ferric HRP has a water ligand. To estimate the coherent response of the five-coordinate ferric HRP, we used the coherence spectrum of native HRP in Figure 6B, probed at $405 \mathrm{~nm}$, which optimizes resonance with the five-coordinate ferric HRP species. (Here we assume that the shoulder near $390 \mathrm{~nm}$ in HRP represents the five-coordinate ferric material, as seen for the five-coordinate H64L metMb spectrum in Figure 1C.) In addition, we also present the coherence spectra of ferric HRPNO at $426 \mathrm{~nm}$ in Figure 6B to probe for the presence of residual hot, six-coordinate, unphotolyzed material arising when the photolysis quantum yield is less than unity. ${ }^{49}$ The differences between the spectra at $405 \mathrm{~nm}$ and the spectra at $426 \mathrm{~nm}$ indicate that such material is likely present.

To further assess the impact of the strong hydrogen bond on the low-frequency modes of ferric HRP, we carried out a comparative measurement between the ferric species of native HRP, metMb, and $\mathrm{Cgb}$, as presented in Figure 7. As seen in the ferrous and ferricyanide forms (Figure 4 and Figure 5), the activity below $\sim 50 \mathrm{~cm}^{-1}$ in ferric HRP is strongly suppressed with respect to metMb. On the other hand, despite the strong hydrogen bond(s) present in $\mathrm{Cgb},{ }^{36}$ there is still an intense low-frequency mode that appears at $34 \mathrm{~cm}^{-1}$, slightly lower than the frequency of the dominant oscillation seen in ferric $\mathrm{Mb}$. This suggests that the hydrogen bonding is not responsible for the dramatic suppression of the modes below $\sim 50 \mathrm{~cm}^{-1}$ in native HRP. This is also consistent with the observation of a moderately intense mode near $37-39 \mathrm{~cm}^{-1}$ when BHA is added to HRP (Figure 3). Stabilization of the distal water ligand by BHA presumably leaves the proximal hydrogen bond intact, which suggests that the modes below $\sim 50 \mathrm{~cm}^{-1}$ are not particularly sensitive to the proximal hydrogen bonding.

The possibility that the ligand charge state might influence the low-frequency sprectrum was also examined. For example, hydrogen bonding that increases the imidazolate character of the proximal histidine ligand could, in principle, reduce the inherent positive charge of the ferric heme and potentially account for some of the spectral differences seen in Figure 4 and Figure 5. To test for the effect of ligand charge, we carried out comparative measurements between metMb and met $\mathrm{MbF}^{-}$, because the negatively charged fluoride ion should more completely neutralize the positively charged ferric heme. The low-frequency FCS spectra of the two species are presented in Figure 8. Although there are some subtle changes in the power spectra, the dominant low-frequency mode at $39 \mathrm{~cm}^{-1}$ is not affected by the presence of the negatively charged $\mathrm{F}^{-}$ion.

Figure 9A shows the FCS spectra of ferrous HRP as a function of temperature. The frequencies noted in the figure are averages over the temperature-dependent data for the separate regions above and below the glass transition at $T_{\mathrm{g}} \sim 180 \mathrm{~K}$. These data were obtained in a sequence of experiments that used identical laser pulses $(430 \mathrm{~nm}, 60 \mathrm{fs})$. Due to the additional noise introduced by the spinning lens apparatus, the frequencies above $200 \mathrm{~cm}^{-1}$ have somewhat decreased amplitudes. However, there are significant effects that are observed when the temperature is lowered. The most striking observation is that the coherence signal persists over much longer timescales at the lowest temperatures. Figure 9B shows a plot of the extracted damping factors as a function of temperature. 


\section{Discussion}

\section{Comparison of HRP and Mb}

In this work, we use femtosecond coherence spectroscopy to investigate the effect of proteininduced distortions on the low-frequency mode structure of the heme. HRP and Mb have different functions, but a common heme group having axial ligands and an iron oxidation-and spin-state that can be controlled. For example, in order to keep the heme in a well-defined ligand/coordination/spin state, and isolate the geometric distortion effects of the protein matrix, we have utilized the ferricyanide adducts. In this way, the low-frequency coherence spectrum can act as an unambiguous reporter of the effect of the surrounding protein environment on the embedded heme.

As can be seen in Figure 1, the absorption spectra of several analogous species of Mb and HRP match closely (e.g., their Soret peaks fall within $\pm 2 \mathrm{~nm}$ ). On the other hand, the vibrational spectra (both Raman and coherence) of these two proteins show significant differences. In Figure 2 and Figure 3, ferric Mb shows a dominant low frequency mode at $39 \mathrm{~cm}^{-1}$, while native HRP has no activity and ferric HRP/BHA has only moderate activity in this region of the spectrum. Because the comparison of the five- and six-coordinate species has shown that the spectral differences are not the result of ligation state, we assign the differences in the coherence spectra to protein-induced distortions of the heme geometry.

Figure 2 and Figure 4B display the correlation between frequency and time domain measurements for the ferric species. As can be seen, there is excellent agreement between the two spectroscopic approaches. There is a certain degree of congestion in the Raman spectra of native HRP (Figure 2B) and HRP/BHA (Figure 2C), which can make the interpretation of the data difficult. The blue shoulder in the absorption spectrum, shown in Figure 1, suggests that the native HRP sample involves a mixture of species. Changes in the Soret bands and the highfrequency Raman spectra ${ }^{30,42}$ upon the addition of BHA to ferric HRP indicate that the fivecoordinate population is removed and the six-coordinate water-bound species is stabilized (e.g., the Soret absorption shows the disappearance of the blue side shoulder near $390 \mathrm{~nm}$ ).

It is worth recalling that some out-of-plane modes are intensity-enhanced in the Raman spectra of HRP (see Figure 2B,C). The band at $693 \mathrm{~cm}^{-1}$, on the shoulder of the $v_{7}$ mode, is assigned to $\gamma_{15}$ with $B_{2 u}$ symmetry, whereas the mode at $716 \mathrm{~cm}^{-1}$ is assigned to $\gamma_{11}$ with $B_{1 u}$ symmetry. 50 These two modes can be made Raman active by the saddling $\left(\mathrm{B}_{2 \mathrm{u}}\right)$ and ruffling $\left(\mathrm{B}_{1 \mathrm{u}}\right)$ heme distortions observed in the $\mathrm{X}$-ray structures (vide infra). There is also an additional shoulder of $\gamma_{11}$ seen in HRP, but not in HRP/BHA, which is assigned to the $\mathrm{A}_{2 \mathrm{u}}$-type $\gamma_{5}$ mode. Because $\gamma_{5}$ can be enhanced by doming displacement, the absence of $\gamma_{5}$ in HRP/BHA may be indicating that the heme is less domed when water is bound to the heme iron.

There are significant changes between the coherence spectra of the HRP species with and without the addition of BHA when the data at $418 \mathrm{~nm}$ are compared in Figure 3. In contrast, there is little difference between the Raman spectra of HRP and HRP/BHA in Figure 2B,C, with the notable exception of a dramatic intensity loss of the $v_{15}$ mode at $750 \mathrm{~cm}^{-1}$ when BHA is bound. The $v_{15}$ mode is depolarized $\left(\mathrm{B}_{1 \mathrm{~g}}\right)$ and has been reported to be either strongly JahnTeller active in the Soret band ${ }^{51}$ or enhanced through a vibronic coupling mechanism. ${ }^{52}$ The Raman spectra of metMb and its H64L mutant, shown in the upper traces of Figure 2A, demonstrate that this mode is quite sensitive to the presence of the water ligand. By analogy, we suggest that the loss of intensity for this mode in Figure $2 \mathrm{C}$ reflects the stabilization of the heme-bound water molecule when BHA is added to HRP. Both the FCS data and the changes in the $750 \mathrm{~cm}^{-1}$ mode Raman intensity demonstrate that BHA is binding close to the heme and altering its vibrational landscape by perturbing its local geometry and stabilizing a bound water ligand. Most of the mode frequencies in the coherence spectra shown in Figure 3 are roughly 
independent of the presence of BHA, but their relative intensities are significantly changed, presumably due to alterations of the relative magnitudes of the protein-induced heme structural distortions (along the out-of-plane heme normal modes).

One clear exception to the frequency correlations between HRP and HRP/BHA is the disappearance of the $144 \mathrm{~cm}^{-1}$ mode and the appearance of a new feature at $170 \mathrm{~cm}^{-1}$ when BHA is added (compare second and third panels in Figure 3). Based on the fact that the heme iron is mostly six-coordinate when BHA is present, ${ }^{30,42}$ it is tempting to assign the 144 $\mathrm{cm}^{-1}$ mode to the five-coordinate material present in the native HRP sample. However, because the coherence spectrum of HRP/BHA taken at $405 \mathrm{~nm}$ (4th panel of Figure 3) clearly shows an oscillation at $143 \mathrm{~cm}^{-1}$, a definitive assignment cannot be made. It is noteworthy that the $144 \mathrm{~cm}^{-1}$ mode does not appear in the FCS spectrum of native HRP measured at $405 \mathrm{~nm}$ (Figure 3 , fifth panel), which should be strongly resonant with the five-coordinate material. The assignments of the low-frequency modes in HRP are clearly problematic. However, this is not too surprising, because the assignment of the better characterized high-frequency Raman modes also has a complicated history. ${ }^{29,42,45}$

\section{Protein-Induced Heme Distortion}

As a result of the complexity associated with the wide variety of possible HRP ligation and spin states, we turned to the ferricyanide adducts to isolate the protein-induced structural perturbations and obtain a more direct comparison between the $\mathrm{Mb}, \mathrm{Cgb}$, and HRP samples (see Figure 4). When $\mathrm{CN}$ is bound, the coordination and spin-states (six-coordinate, low-spin) are identical. Nevertheless, the FCS spectra are still very different, with the HRPCN spectrum dominated by a strong mode at $96 \mathrm{~cm}^{-1}$, while $\mathrm{MbCN}$ shows a major mode near $40 \mathrm{~cm}^{-1}$. The $\mathrm{CbgCN}$ spectrum displays two strong modes at $30 \mathrm{~cm}^{-1}$ and $79 \mathrm{~cm}^{-1}$. Given the X-ray structures discussed below, we tentatively assign the strong $96 \mathrm{~cm}^{-1}$ in HRPCN to saddling and the mode near $79 \mathrm{~cm}^{-1}$ in $\mathrm{CgbCN}$ to ruffling. The modes at $40 \mathrm{~cm}^{-1}$ in $\mathrm{MbCN}$, and at 30 $\mathrm{cm}^{-1}$ in $\mathrm{CgbCN}$, are consistent with the heme doming assignment. However, there is a possible exception in the $\mathrm{Cgb}$ doming assignment because there is only a small doming displacement and the phase of the $30 \mathrm{~cm}^{-1}$ mode is found to be near zero. The phase suggests a field driven coordinate displacement for the wavepacket, which is distinctly different than what is observed for $\mathrm{MbCN} .{ }^{41}$ Thus, it is possible that the mode between 30 and $34 \mathrm{~cm}^{-1}$, seen in all $\mathrm{Cgb}$ samples, represents an additional unassigned low-frequency heme motion.

The ferrous forms of $\mathrm{Mb}, \mathrm{HRP}$, and $\mathrm{Cgb}$ also have well-defined ligation, oxidation, and spin states. The Fe-His stretching modes of these proteins appear at very different positions (219, 248 , and $253 \mathrm{~cm}^{-1}$, respectively). As noted previously, the increase in the frequency of this mode in HRP is attributed ${ }^{53}$ to the strong hydrogen bond that the proximal histidine (His170) establishes with an adjacent amino acid (Asp247). The hydrogen bond confers an imidazolate (negatively charged) character to the proximal ligand and leads to a stronger bond between the heme iron and His $170,{ }^{53}$ which could conceivably result in a larger iron out-of-plane displacement and more distortion of the heme plane. Cbg also has strong hydrogen bonding to the proximal histidine, which is thought to involve two amino acids (Tyr84 and Glu134). ${ }^{36}$

Figure 5 shows that the reduced samples of $\mathrm{Mb}$ and HRP have their dominant vibrational features centered near $100 \mathrm{~cm}^{-1}$ but with two modes for deoxyMb (at 98 and $119 \mathrm{~cm}^{-1}$ ) and with four modes for $\mathrm{HRP}\left(\mathrm{Fe}^{2+}\right.$ ) (at 57, 78, 104, and $128 \mathrm{~cm}^{-1}$ ). The deoxy Mb sample also displays a strong doming mode near $46 \mathrm{~cm}^{-1}$, whereas HRP has only a weak shoulder near 39 $\mathrm{cm}^{-1}$. The origin of the weak feature near $\sim 18 \mathrm{~cm}^{-1}$, seen in the HRP and Mb samples, is unknown and could arise from over- or under-fitting the population response. The ferrous $\mathrm{Cbg}$ sample, on the other hand, displays strong oscillations at 31 and $54 \mathrm{~cm}^{-1}$, with significant additional features at 87,119 , and $142 \mathrm{~cm}^{-1}$. 
The HRP sample shows a distinct lack of low-frequency mode activity below $\sim 50 \mathrm{~cm}^{-1}$ in the ferrous (Figure 5), ferric (Figure 7), and CN-bound (Figure 4) states. To test the possibility that the proximal hydrogen bond might be responsible for this behavior, we turn to the coherence spectra of $\mathrm{Cgb}$, which also has a strong proximal hydrogen bonding network. ${ }^{36}$ The spectra display strong modes between 30 and $34 \mathrm{~cm}^{-1}$ in the ferric, ferrous, and CN-bound $\mathrm{Cgb}$ samples, demonstrating that the suppression of modes below $\sim 50 \mathrm{~cm}^{-1}$ in HRP is not the result of proximal hydrogen bonding. This conclusion is also consistent with the observation of a moderately intense mode near $37-39 \mathrm{~cm}^{-1}$ (with anomalous phase near $-\pi / 2$ at $418 \mathrm{~nm}$ and $\pi / 4$ at $405 \mathrm{~nm}$ ) when BHA is added to HRP (Figure 3). Stabilization of the distal water ligand by BHA presumably leaves the proximal hydrogen bond intact, which suggests that the modes below $\sim 50 \mathrm{~cm}^{-1}$ are not particularly sensitive to the proximal hydrogen bonding.

The possibility that the charge of the axial ligand may influence the low-frequency spectrum was tested by measurements that compared the water-bound metMb to the fluoride-bound adduct, as shown in Figure 8. Although there are minor changes when the two spectra are compared, the mode at $39 \mathrm{~cm}^{-1}$ (with phase $\sim \pi / 2$ ) is the dominant feature in both. We conclude that the ionization state of the heme ligand is not likely to be responsible for the significant changes in the coherence spectra of HRP compared to Mb.

Overall, the comparisons demonstrate that neither H-bonding of the proximal histidine in native HRP nor its imidazolate nature are responsible for the reduction in amplitude of the modes below $\sim 50 \mathrm{~cm}^{-1}$, which have been found between 27 and $44 \mathrm{~cm}^{-1}$ in most other ferric heme systems. ${ }^{37,39,41}$ A more likely explanation is related to the lowered heme symmetry that results from specific protein-induced geometric distortions. The symmetry of the heme controls which specific modes are allowed in the coherence spectra. The heme is generally planar when it is unconstrained by the protein matrix but, when inserted into a protein, it can interact at various levels with the surrounding amino acids (steric, hydrogen bonds, van der Waals forces, etc.). These interactions translate into distortions of the heme geometry along the "soft" lowfrequency modes that can alter the heme symmetry group and result in the enhancement of the out-of-plane mode intensity in the coherence spectrum (M. Kubo et al., to be published).

There are X-ray structure reports available for many Mb and HRP species, ${ }^{44,54,55}$ and comparisons of the various derivatives of $\mathrm{Mb}, \mathrm{Cgb}$, and $\mathrm{HRP}$ are presented in Figure 10 (the only X-ray structure of $\mathrm{Cgb}$ currently available is the cyanide adduct from the laboratory of R. K. Poole, which will be published elsewhere). Although the axial ligands, spin, oxidation, and coordination states are the same when either the ferrous forms or the ferricyanide derivatives are compared, the structure of the heme in the various protein environments is very different. In Figure 10, we present a NSD analysis, which is useful to quantify the protein-induced heme deformations (see below). While the dominant relative distortion of the heme in myoglobin appears to be doming, the heme in HRP is significantly saddled and much less domed. ${ }^{34,45}$ Figure 10 shows the structure of the heme in HRPCN, but similar distortions persist in the other ferric forms of HRP. It should be noted that the heme of $\mathrm{CgbCN}$ is only moderately saddled and ruffled in comparison to HRP. Finally, it can be seen that the propionic side chains for the HRP samples are in an eclipsed geometry, while those of the globins are staggered. We suggest that these protein-induced heme distortions lead to symmetry reduction and control the coupling associated with low-frequency mode intensity in the coherence spectra of these three different proteins.

\section{Photolysis Reactions}

We have measured the coherence spectra of both the ferrous and ferric NO-bound species of HRP, and the results are presented in Figure 6 and Table 2. The main observation is the appearance of a strong low-frequency mode near $40 \mathrm{~cm}^{-1}$ in both species, which is especially dominant in the ferric sample. Both species are highly photolabile and undergo photolysis, 
with quantum yields similar to the corresponding Mb species. ${ }^{48}$ The doming mode is strongly coupled to the photolysis process, and there have been a number of previous studies ${ }^{37,39,56}$ that locate the doming mode in heme proteins near $\sim 40 \mathrm{~cm}^{-1}$. We previously observed a $\sim 40$ $\mathrm{cm}^{-1}$ doming mode that is enhanced by photolysis for MbNO.${ }^{57}$ Even though HRP does not appear to have an active doming mode in its resting state, a significant transient doming deformation upon photolysis is demonstrated by the experimental observations reported here (Figure 6).

Overall, a detailed comparison of the low-frequency heme modes of Mb and HRP leads to the consistent observation that HRP does not reveal coupling to modes below $\sim 50 \mathrm{~cm}^{-1}$ unless BHA is bound (stabilizing the water ligand) or photolysis is involved. On the other hand, all the $\mathrm{Mb}$ species have strong coherent oscillations that appear near $40 \mathrm{~cm}^{-1}$. The use of the $\mathrm{CN}$ bound species allows $\mathrm{Mb}$ and HRP to be compared and demonstrates that the observed differences in the low-frequency coherence spectra must be related to either the protein-induced distortions of the heme geometry or transient distortions associated with photolysis. The protein-induced distortions may have functional significance, especially if the distorted lowfrequency modes participate as reaction coordinates.

\section{Normal Coordinate Structural Decomposition}

NSD has been used previously to quantify the role of heme out-of-plane deformations. ${ }^{34,58 \text {, }}$ 59 The NSD method can be implemented by using the lowest frequency out-of-plane normal coordinates of each symmetry type to simulate the observed structural distortions of the heme group that are observed by X-ray crystallography. Although such calculations are simplified and only use the heme core to form the normal mode basis set, this type of analysis provides a valuable guide to the interpretation of the low-frequency coherence spectra of the heme group. As shown in Figure 10, linear combinations of the main deformation modes (saddling, ruffling, doming, inverse doming, waving ${ }_{\mathrm{x}}$, waving $\mathrm{y}$, and propellering) are able to describe the nonplanar heme structures in proteins under investigation here, as well as most of the published $\mathrm{X}$-ray structural data on heme proteins and synthetic metalloporphyrins. ${ }^{34,59-62}$

For example, an examination of the frequencies observed in the coherence spectra of the ferric samples of $\mathrm{Mb}$ and $\mathrm{HRP}$ (Table 1 and Table 2) results in an almost one-to-one correspondence (within $\pm 5 \mathrm{~cm}^{-1}$ ), with differences appearing primarily in the relative amplitudes of the specific modes. This behavior supports the idea that symmetry-breaking deformations of the heme are induced to various degrees that are specific to each protein. The specific frequencies predicted by density functional theory (DFT) calculations for each low-frequency out-of-plane deformation mode correlate extremely well with the experimental data we have obtained for porphine (M. Kubo et al., to be published), which strongly supports the mode assignments presented here. However, the DFT correlations are more difficult to ascertain when side chains and axial ligands are added to the chromophore. This is because the resulting vibrational normal modes become complex mixtures of the porphine basis states plus the side chain and ligand motions. Nevertheless, the protein-specific heme structural distortions, as quantified using the porphine deformation modes as a basis, can be used to both assign and predict variations in the protein-specific low-frequency vibrational spectra.

It turns out that as the protein deformation of the heme becomes larger along a given normal coordinate, the amplitude of that specific mode in the coherence spectrum increases with the square of the distortion (M. Kubo, to be published). For example, the largest heme deformation typically found in $\mathrm{Mb}$ (from the NSD analysis) is represented by doming. ${ }^{34}$ Thus, an examination of the coherence spectrum of this species leads us to assign the features near 40 $\mathrm{cm}^{-1}$ in metMb, MbCN, and deoxyMb to the doming mode. This result supports previous reports ${ }^{37,39,56}$ that have placed the doming mode in heme proteins in the region [37-47 $\mathrm{cm}^{-1}$ ]. The same analysis can be carried out for other heme species and careful comparisons 
between structure deformations and coherence spectra, using a larger experimental database, leads to improved assignments of low-frequency modes (M. Kubo et al., to be published). The analysis so far indicates that, for modes below $\sim 100 \mathrm{~cm}^{-1}$, there is a direct relationship between the NSD components that describe the nonplanar heme structure distortions (such as doming, ruffling, and saddling that are induced by the different proteins) and the specific low-frequency mode patterns that become active in the coherence spectra. For example, using this type of analysis, we have proposed that the mode at $96 \mathrm{~cm}^{-1}$ in HRPCN is activated by heme saddling and that the mode near $79 \mathrm{~cm}^{-1}$ in $\mathrm{CgbCN}$ is due to heme ruffling (see Figure 10).

\section{Functional Roles and Dephasing}

These same heme distortions may also correlate with specific functional roles, as has already been shown for the doming mode and CO binding. ${ }^{28,63,64}$ For example, heme ruffling has been suggested ${ }^{65}$ to be a distortion that is involved in stabilizing the net positive charge of the ferric heme in nitrophorin (NP4). The general picture that needs to be explored is whether or not the protein matrix interacts with the normally planar heme and causes it to adopt conformations that enhance or retard specific reactions. This type of protein control mechanism may apply to reactions other than diatomic ligand binding, such as electron transfer. Clearly, the $\mathrm{d}$ - and $\pi$ orbital overlaps of the iron-porphyrin system will be affected by out-of-plane geometric distortions, and this can affect a variety of its electronic and redox properties. It remains an open question if such reactions are coupled to low-frequency heme modes and can be influenced by protein-induced heme distortions. Protein-based tuning of the heme geometry (and its corresponding electronic properties) could turn out to be one of the mechanisms that allows a single chemical entity (i.e., the heme group) to perform such an impressive array of biological functions. As pointed out previously, ${ }^{34,66}$ certain key heme distortions appear to be conserved across proteins with similar functionality from different species. Peroxidases, for example, have highly conserved characteristic distortions even when they have a large natural variance in the amino acid sequence. ${ }^{34}$

In addition to having the potential to induce functionally important heme distortions, the surrounding protein material evidently also plays a role in screening the chromophore from solvent fluctuations. It has been shown that the low-frequency coherent oscillations of various heme systems persist for longer timescales when the heme is embedded in a protein matrix. ${ }^{67}$ Generally, the damping of the coherence signal has three main sources: spectral inhomogeneity, vibrational lifetime, and "pure" dephasing (coupling to the fluctuations and phonon scattering of the surroundings). Low-frequency out-of-plane modes have relatively long periods of oscillation ( $0.8 \mathrm{ps}$ for the $40 \mathrm{~cm}^{-1}$ mode) and they involve large masses and engage substantial segments of the chromophore. Moreover, as discussed above, lowfrequency modes are likely candidates for biological reaction coordinates. Recent work has suggested that such modes may even have quantum coherent aspects that are functional. ${ }^{56,68}$ If reactions proceed through the sequential activation of such modes, a protection mechanism would be advantageous because it would allow additional time for the coherence to propagate.

\section{Thermal Effects}

The general effect of the solvent "glass" transition, at temperature $T_{\mathrm{g}} \sim 180 \mathrm{~K}$ (for $75 \%$ (v/v) glycerol water mixtures), has been studied in prior work on heme proteins and heme model compounds. ${ }^{28,69-75}$ To further investigate the interactions between the heme chromophore and its surroundings, we carried out a series of temperature-dependent FCS measurements on the ferrous HRP species. We have reported similar FCS studies on deoxy $\mathrm{Mb}^{40}$ and the current data is consistent with the earlier work. As discussed above, the results in Figure 9 show that the damping time of the coherent signal is increased as the temperature is decreased. The coherence spectra are dominated by four major modes having average frequencies of 57, 78, 99, and $124 \mathrm{~cm}^{-1}$ for $T>T_{\mathrm{g}}$, and 54, 74, 93, and $119 \mathrm{~cm}^{-1}$ for $T<T_{\mathrm{g}}$ (see Table 4). The mode 
frequencies display a marked decrease as the system passes through the glass transition near $T_{\mathrm{g}} \sim 180 \mathrm{~K}$. This suggests that the protein-chromophore system responds to structural changes induced by solvent glass transition. Below the glass transition, the system is constrained and the mode frequencies display a small increase as the temperature continues to decrease toward $10 \mathrm{~K}$. Upshifts in frequency as temperature is decreased below $T_{\mathrm{g}}$ have been reported previously for the $\mathrm{Fe}-\mathrm{His}$ mode in deoxyMb $\mathrm{b}^{75-77}$ and can be explained if we assume that anharmonicity is present in the heme potential. $57,70,78$

The increase in the damping time of coherence signals at low temperature is more evident when the temperature is lowered below the glass transition of the solvent $(\sim 180 \mathrm{~K})$. It is particularly apparent at the lowest temperatures in Figure 9, where oscillatory activity with significant amplitude can be observed beyond $4 \mathrm{ps}$. The temperature dependence of the damping time for each mode is plotted in Figure 9B, and it can be seen that the dephasing time begins to dramatically increase below $\sim 50 \mathrm{~K}$. This suggests that the phonons responsible for the "pure" dephasing behavior lie at, or below, $\sim 30 \mathrm{~cm}^{-1}$. It would be of obvious interest to probe the temperature dependence of the bare heme model compound to determine if the dephasing phonons are related in some way to the presence of protein material, which is known to have a peak in its vibrational density-of-states near $\sim 20 \mathrm{~cm}^{-1} .{ }^{79}$

The fact that the dephasing times continue to lengthen at temperatures below the solvent glass transition supports the hypothesis that pure dephasing (rather than inhomogeneity) represents a major source of damping of the low-frequency oscillations in heme protein systems. It also appears that we can rule out inhomogeneity as a source of the more rapidly damping coherences in model compounds at room temperature. ${ }^{67}$ Recent kinetic studies have revealed a significant amount of inhomogeneity that is inherent to the heme group in solution, but it appears that the inhomogeneous distributions are some-what larger in the protein. ${ }^{28}$ Further experimental testing of these ideas will require temperature-dependent measurements of the coherence spectra of heme model compounds.

\section{Summary}

The native HRP and HRPCN species display suppressed coherent activity below $\sim 50 \mathrm{~cm}^{-1}$. In contrast, both ferric HRPNO and HRP/BHA display modes near $40 \mathrm{~cm}^{-1}$ that are associated with heme doming. The $\sim 40 \mathrm{~cm}^{-1}$ mode of ferric HRPNO is evidently activated by the photolysis of the NO ligand, and a smaller but similar effect is seen in the corresponding NObound ferrous species. The moderate activity of the mode near $37-39 \mathrm{~cm}^{-1}$ for HRP/BHA is not completely understood. However, it could indicate the presence of a small heme doming distortion, which was not detected in the X-ray structure ${ }^{80}$ (it should be noted that X-ray photoreduction can lead to complications in the analysis of ferric heme protein X-ray structures). Another possibility is that the water ligand undergoes photolysis in the HRP/BHA complex (and possibly the metMb complex as well). The phase of the $37-39 \mathrm{~cm}^{-1}$ mode in HRP/BHA is found to be $\sim-\pi / 2$ at $418 \mathrm{~nm}$ and $\sim \pi / 4$ at $405 \mathrm{~nm}$, which indicates that photolysis, or some other nonradiative process, leads to significant momentum transfer to the initial wavepacket.

The low-frequency response of native HRP and HRPCN contrasts with that of other heme proteins and heme model compounds, which show strong low-frequency modes in the 27-44 $\mathrm{cm}^{-1}$ range. ${ }^{41}$ Comparisons with $\mathrm{Cgb}$ and metMbF${ }^{-}$rule out hydrogen bonding of the proximal histidine (His 170) or charge imbalance due to its imidazolate character as the source of this unusual behavior. On the other hand, we have recently suggested that the proximal histidine (His93) of MbCN undergoes photolysis with a relatively high ( $75 \%)$ quantum yield. ${ }^{41}$ This process results in the large amplitude and anomalous phase $( \pm \pi / 2)$ observed for the doming mode appearing near $40 \mathrm{~cm}^{-1}$ in $\mathrm{MbCN} .{ }^{41}$ If hydrogen bonding prevents the proximal histidine 
photolysis reaction from taking place, we would expect both HRP and Cgb to have very weak features in the doming region between 30 and $50 \mathrm{~cm}^{-1}$. However, if the intense mode at 30 $\mathrm{cm}^{-1}$ in $\mathrm{CgbCN}$ turns out to be the doming mode, it means that $\mathrm{H}$-bonding cannot be invoked as the reason for the suppression of doming activity in the HRPCN sample. Moreover, the presence of a similar pattern of suppressed amplitude in the modes below $50 \mathrm{~cm}^{-1}$ for ferrous HRP compared to ferrous $\mathrm{Mb}$, where photolysis is not taking place (Figure 5), reinforces the conclusion that a difference in protein-induced heme distortion (Figure 10) is the overarching reason for the contrasting behavior of HRP and Mb.

In summary, we have demonstrated that protein-specific environments, which alter the structure of the heme prosthetic group, can be probed by femtosecond coherence spectroscopy. Protein-induced tuning of the heme out-of-plane distortions can affect, and potentially control, a variety of protein functions. ${ }^{28,65}$ Normal coordinate structural decomposition, when combined with experimental results such as those presented here, shows promise as a method to assign specific, functionally important, low-frequency heme motions. For the most part, the amplitudes of the out-of-plane distortions observed using X-ray crystallography appear to correlate well with the amplitudes of the modes that are active in the coherence spectra. Lowering the temperature has a clear effect on the coherence frequencies and downshifts are observed as the sample passes through the solvent glass transition. These results, along with the comparisons between HRP, Mb, and Cgb, demonstrate that the heme out-of-plane modes are sensitive to the constraints mandated by specific protein environments. The longer coherence dephasing times that appear as the temperature is lowered below $50 \mathrm{~K}$ suggest that pure dephasing, associated with phonon modes near $30 \mathrm{~cm}^{-1}$, represents a major source of damping of the low-frequency oscillations in heme protein systems. In the absence of protein material, the damping of the coherent motion takes place on a significantly faster time scale. 67

\section{Acknowledgments}

This work is supported by grants from NIH (DK035090) and NSF (0211816) to P.M.C., NIH (HL65465) to S.-R.Y., and BBSRC (D18084) to R.K.P. M.K. thanks JSPS for a postdoctoral fellowship for research abroad.

\section{References}

1. Antonini, E.; Brunori, M. Hemoglobin and myoglobin in their reactions with ligands. AmsterdamLondon: North-Holland Publishing Co; 1971.

2. Bredt DS, Snyder SH. Annu. Rev. Biochem 1994;63:175-195. [PubMed: 7526779]

3. Dawson TM, Snyder SH. J. Neurosci 1994;14:5147-5159. [PubMed: 8083727]

4. Dickerson, RE.; Geis, I. Hemoglobin: Structure, Function, Evolution, and Pathology. Menlo Park, CA: The Benjamin/Cummings Publishing Company, Inc; 1983.

5. Dunford, HB. Heme peroxidases. New York: John Wiley; 1999.

6. Kincaid, J. Resonance Raman Spectra of Heme Proteins and Model Compounds. In: Kadish, KM.; Smith, KM.; Guilard, R., editors. The Porphyrin Handbook. New York: Academic Press; 2000. p. 225-292.

7. Moore, GR.; Pettigrew, GW. Cytochromes c. Evolutionary, Structural and Physicochemical Aspects. Berlin, Heidelberg, New York, London, Paris, Tokyo, Hong Kong, Barcelona: Springer-Verlag; 1990. p. 478

8. Ortiz de Montellano, PR. Cytochrome P450: Structure, mechanism, and biochemistry. New York: Plenum Press; 1995.

9. Scott, RA.; Mauk, AG. Cytochrome C: A Multidisciplinary Approach. Sausalito: University Science Books; 1996.

10. Aono S. Acc. Chem. Res 2003;36:825-831. [PubMed: 14622029]

11. Singh S, Madzelan P, Banerjee R. Nat. Prod. Rep 2007;24:631-639. [PubMed: 17534535] 
12. Dawson JH, Pond AE, Roach MP. Biopolymers 2002;67:200-206. [PubMed: 12012432]

13. Howes BD, Heering HA, Roberts TO, Schneider-Belhadadd F, Smith AT, Smulevich G. Biopolymers 2001;62:261-267. [PubMed: 11745121]

14. Rohlfs RJ, Mathews AJ, Carver TE, Olson JS, Springer BA, Egeberg KD, Sligar SG. J. Biol. Chem 1990;265:3168-3176. [PubMed: 2303446]

15. Barkigia KM, Chantranupong L, Smith KM, Fajer J. J. Am. Chem. Soc 1988;110:7566-7567.

16. Ravikanth M, Chandrashekar TK. Struct. Bonding (Berlin) 1995;82:105-188.

17. Senge, M. The Porphyrin Handbook. Kadish, KM.; Smith, KM.; Guilard, R., editors. Boston: Academic Press; 2000. p. 239-248.

18. Shelnutt JA, Song XZ, Ma JG, Jia SL, Jentzen W, Medforth CJ. Chem. Soc. Rev 1998;27:31-41.

19. Fleming GR, Joo T, Cho M, Zewail AH, Letokhov VS, Marcus RA, Pollak E, Tannor DJ, Mukamel S. Adv. Chem. Phys 1997;101:141-183.

20. Jonas DM, Bradforth SE, Passino SA, Fleming GR. J. Phys. Chem 1995;99:2594-2608.

21. Kumar ATN, Rosca F, Widom A, Champion PM. J. Chem. Phys 2001;114:701-724.

22. Kumar ATN, Rosca F, Widom A, Champion PM. J. Chem. Phys 2001;114:6795-6815.

23. Mukamel, S. Principles of Nonlinear Optical Spectroscopy. New York: Oxford University Press; 1995.

24. Pollard WT, Dexheimer SL, Wang Q, Peteanu LA, Shank CV, Mathies RA. J. Phys. Chem 1992;96:6147-6158.

25. Shen, YR. The Principles of Nonlinear Optics. New York: John Wiley \& Son; 1984. p. 141-186.

26. Zewail, AH. Femtochemistry: Ultrafast Dynamics of the Chemical Bond. Singapore: World Scientific Publishing Co. Pte. Ltd; 1994.

27. Ziegler LD, Fan R, Desrosiers AE, Scherer NF. J. Chem. Phys 1994;100:1823-1839.

28. Ye, Ionascu D, Gruia F, Yu A, Champion P. Proc. Natl. Acad. Sci. U.S.A 2007;104:14682-14687. [PubMed: 17804802]

29. Evangelista-Kirkup R, Crisanti M, Poulos TL, Spiro TG. FEBS Lett 1985;190:221-226. [PubMed: 2995134]

30. Smulevich G, Feis A, Indiani C, Becucci M, Marzocchi MP. JBIC, J. Biol. Inorg. Chem 1999;4:39_ 47.

31. Smulevich G, Feis A, Howes BD. Acc. Chem. Res 2005;38:433-440. [PubMed: 15895981]

32. Veitch NC. Phytochemistry 2004;65:249-259. [PubMed: 14751298]

33. Elvers KT, Wu G, Gilberthorpe NJ, Poole RK, Park SF. J. Bacteriol 2004;186:5332-5341. [PubMed: 15292134]

34. Jentzen W, Ma JG, Shelnutt JA. Biophys. J 1998;74:753-763. [PubMed: 9533688]

35. Champion PM. Science 2005;310:980-982. [PubMed: 16284167]

36. Lu C, Mukai M, Lin Y, Wu G, Poole RK, Yeh SR. J. Biol. Chem 2007;282:25917-25928. [PubMed: 17606611]

37. Rosca F, Kumar ATN, Ye X, Sjodin T, Demidov AA, Champion PM. J. Phys. Chem 2000;104:42804290.

38. Constantine S, Zhou Y, Morais J, Ziegler LD. J. Phys. Chem. A 1997;101:5456-5462.

39. Wang W, Ye X, Demidov AA, Rosca F, Sjodin T, Cao W, Sheeran M, Champion PM. J. Phys. Chem. B 2000;104:10789-10801.

40. Ionascu D, Rosca F, Gruia F, Yu A, Champion P. Rev. Sci. Instrum 2006;77

41. Gruia F, Kubo M, Ye X, Champion P. Biophys. J 2008;94:2252-2268. [PubMed: 18065461]

42. Smulevich G, Paoli M, Burke JF, Sanders SA, Thorneley RN, Smith AT. Biochemistry 1994;33:73987407. [PubMed: 8003505]

43. Cao W, Christian JF, Champion PM, Rosca F, Sage JT. Biochemistry 2001;40:5728-5737. [PubMed: 11341838]

44. Quillin ML, Arduini RM, Olson JS, Phillips GN Jr. J. Mol. Biol 1993;234:140-155. [PubMed: 8230194] 
45. Howes BD, Schiodt CB, Welinder KG, Marzocchi MP, Ma JG, Zhang J, Shelnutt JA, Smulevich G. Biophys. J 1999;77:478-492. [PubMed: 10388773]

46. Kitagawa, T. Heme protein structure and the iron histidine stretching mode. In: Spiro, TG., editor. Biological Applications of Raman Spectroscopy. New York, Chichester, Brisbane, Toronto, Singapore: Wiley-Interscience Publication; 1988. p. 97-131.

47. Howes BD, Feis A, Raimondi L, Indiani C, Smulevich G. J. Biol. Chem 2001;276:40704-40711. [PubMed: 11546788]

48. Ye X, Yu A, Champion PM. J. Am. Chem. Soc 2006;128:1444-1445. [PubMed: 16448103]

49. Ye X, Demidov AA, Champion PM. J. Am. Chem. Soc 2002;124:5914-5924. [PubMed: 12010067]

50. Li D, Stuehr DJ, Yeh SR, Rousseau DL. J. Biol. Chem 2004;279:26489-26499. [PubMed: 15066989]

51. Shelnutt JA, Cheung LD, Chang RCC, Yu NT, Felton RH. J. Chem. Phys 1977;66:3387-3398.

52. Hu S, Morris IK, Singh JP, Smith KM, Spiro TG. J. Am. Chem. Soc 1993;115:12446-12458.

53. Teraoka J, Kitagawa T. J. Biol. Chem 1981;256:3969-3977. [PubMed: 7217068]

54. Berglund GI, Carlsson GH, Smith AT, Szoke H, Henriksen A, Hajdu J. Nature 2002;417:463-468. [PubMed: 12024218]

55. Gajhede M, Schuller DJ, Henriksen A, Smith AT, Poulos TL. Nat. Struct. Biol 1997;4:1032-1038. [PubMed: 9406554]

56. Liebl U, Lipowski G, Negrerie M, Lambry JC, Martin JL, Vos MH. Nature 1999;401:181-184. [PubMed: 10490029]

57. Rosca F, Kumar ATN, Ionascu D, Ye X, Demidov AA, Sjodin T, Wharton D, Barrick D, Sligar SG, Yonetani T, Champion PM. J. Phys. Chem. A 2002;106:3540-3552.

58. Ma JG, Zhang J, Franco R, Jia SL, Moura I, Moura JJ, Kroneck PM, Shelnutt JA. Biochemistry 1998;37:12431-12442. [PubMed: 9730815]

59. Shelnutt JA. J. Porphyrins Phthalocyanines 2001:300-311.

60. Ma JG, Laberge M, Song XZ, Jentzen W, Jia SL, Zhang J, Vanderkooi JM, Shelnutt JA. Biochemistry 1998;37:5118-5128. [PubMed: 9548742]

61. Song XZ, Jentzen W, Jaquinod L, Khoury RG, Medforth CJ, Jia SL, Ma JG, Smith KM, Shelnutt JA. Inorg. Chem 1998;37:2117-2128. [PubMed: 11670364]

62. Song Y, Haddad RE, Jia SL, Hok S, Olmstead MM, Nurco DJ, Schore NE, Zhang J, Ma JG, Smith KM, Gazeau S, Pecaut J, Marchon JC, Medforth CJ, Shelnutt JA. J. Am. Chem. Soc 2005; 127:1179_ 1192. [PubMed: 15669857]

63. Champion PM. J. Raman Spectrosc 1992;23:557-567.

64. Srajer V, Reinisch L, Champion PM. J. Am. Chem. Soc 1988;110:6656-6670.

65. Roberts S, Weichsel A, Qiu Y, Shelnutt J, Walker F, Montfort W. Biochemistry 2001;40:1132711337. [PubMed: 11560480]

66. Hobbs JD, Shelnutt JA. J. Protein Chem 1995;14:19-25. [PubMed: 7779259]

67. Gruia F, Ye X, Ionascu D, Kubo M, Champion P. Biophys. J 2007;93:4404-4413. [PubMed: 17766351]

68. Prokhorenko VI, Nagy AM, Waschuk SA, Brown LS, Birge RR, Miller RJ. Science 2006;313:12571261. [PubMed: 16946063]

69. Austin RH, Erramilli S. Methods Enzymol 1995;246:131-168. [PubMed: 7752923]

70. Bitler A, Stavrov SS. Biophys. J 1999;77:2764-2776. [PubMed: 10545375]

71. Cusack S, Doster W. Biophys. J 1990;58:243-251. [PubMed: 2166599]

72. Indiani C, Feis A, Howes BD, Marzocchi MP, Smulevich G. J. Inorg. Biochem 2000;79:269-274. [PubMed: 10830877]

73. Ionascu D, Gruia F, Ye X, Yu A, Rosca F, Beck C, Demidov A, Olson JS, Champion PM. J. Am. Chem. Soc 2005;127:16921-16934. [PubMed: 16316238]

74. Postlewaite JC, Miers JB, Dlott DD. J. Am. Chem. Soc 1989;111:1248-1255.

75. Sage JT, Schomacker KT, Champion PM. J. Phys. Chem 1995;99:3394-3405.

76. Ondrias MR, Rousseau DL, Simon SR. J. Biol. Chem 1983;258:5638-5642. [PubMed: 6853537]

77. Sassaroli M, Dasgupta S, Rousseau DL. J. Biol. Chem 1986;261:13704-13713. [PubMed: 3759989]

J Am Chem Soc. Author manuscript; available in PMC 2009 November 30. 
78. Korostishevsky M, Zaslavsky Z, Stavrov SS. Biophys. J 2004;86:656-659. [PubMed: 14695309]

79. Go N. Biophys. J 1978;17:1373-1379.

80. Henriksen A, Schuller DJ, Meno K, Welinder KG, Smith AT, Gajhede M. Biochemistry 1998;37:8054-8060. [PubMed: 9609699] 


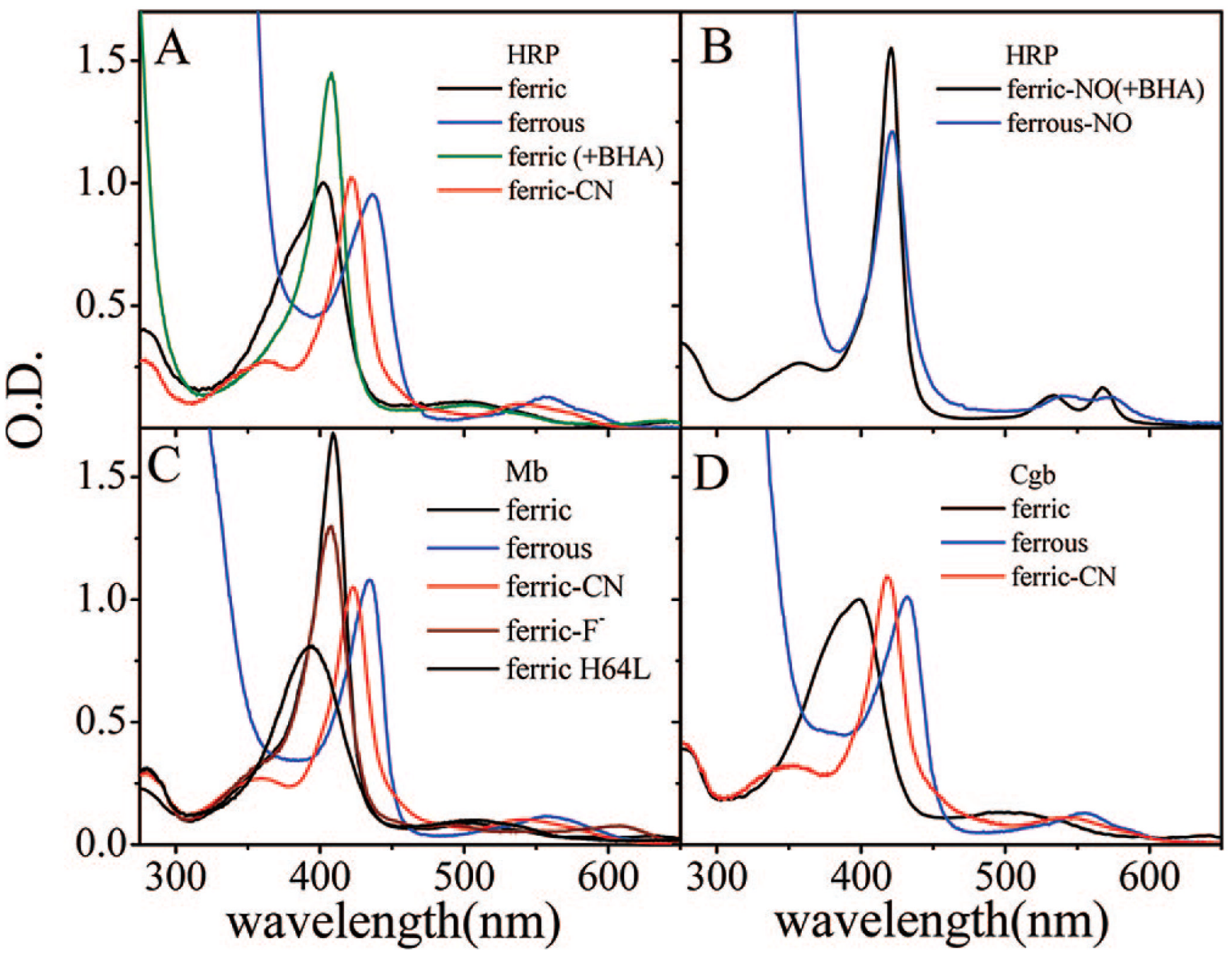

Figure 1.

(A) Electronic absorption spectra of ferric, ferric/BHA, ferrous and cyanide-bound species of HRP. The Soret maxima are located at 402, 408, 437, and $423 \mathrm{~nm}$, respectively. (B) Electronic absorption spectra of ferric and ferrous species of HRP-NO. The Soret maxima are located at $422 \mathrm{~nm}$. (C) Electronic absorption spectra of ferric, ferric H64L, ferric fluoride-bound, ferrous, and cyanide-bound species of Mb measured. The Soret maxima are located at 409, 390, 408, 435 , and $423 \mathrm{~nm}$, respectively. (D) Electronic absorption spectra of ferric, ferrous, and cyanidebound species of Cgb. The Soret maxima are located at 398, 432, and $418 \mathrm{~nm}$, respectively. All spectra in Figure 1 are measured at room temperature. 

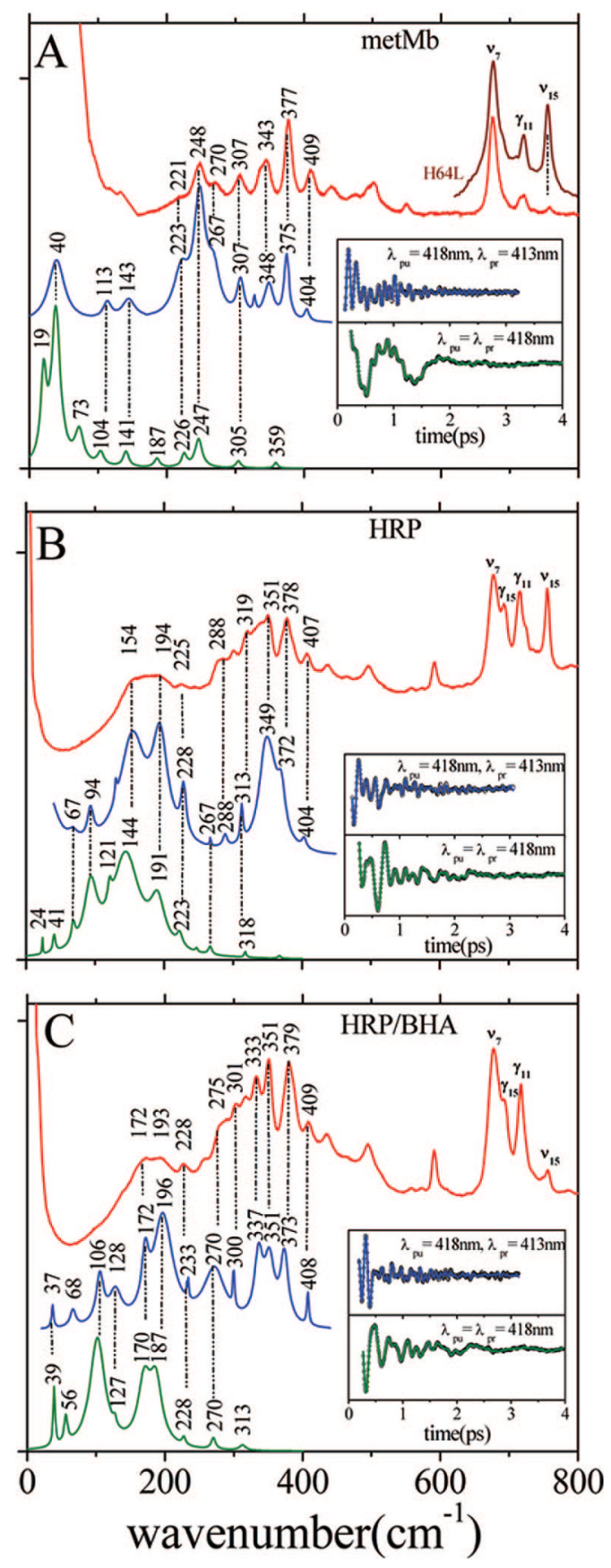

Figure 2.

(A) Correlations between the Raman and coherence spectra of ferric Mb. The Raman spectrum of the five-coordinate $\mathrm{H} 64 \mathrm{~L} \mathrm{Mb}$ mutant in the $600-800 \mathrm{~cm}^{-1}$ region is also displayed to show the strong $v_{15}$ at $750 \mathrm{~cm}^{-1}$. (B) Correlations between the Raman and coherence spectra of native ferric HRP. (C) Correlations between the Raman and coherence spectra of ferric HRP/BHA. The inset shows the coherent oscillations and LPSVD fits corresponding to open band (green) and dispersed (blue) detection. The Raman data were measured with a $413.1 \mathrm{~nm}$ excitation wavelength, while the FCS data were measured using a $418 \mathrm{~nm}$ excitation. The dispersed data were measured with a $0.5 \mathrm{~nm}$ spectral window, detuned $5 \mathrm{~nm}$ to the blue of the carrier frequency $\operatorname{maximum}\left(\lambda_{\mathrm{pr}}=413 \mathrm{~nm}\right)$. 

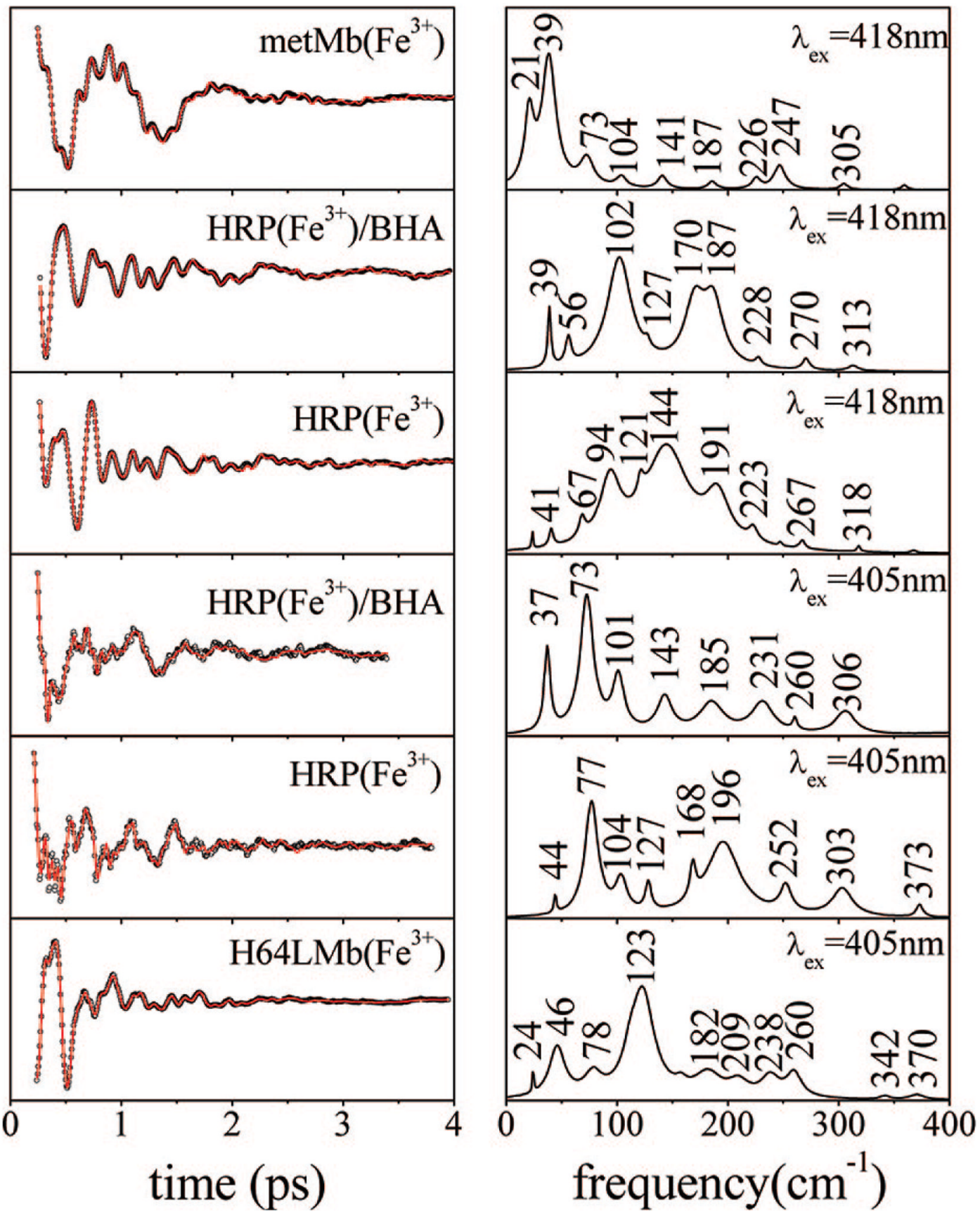

Figure 3.

Coherent oscillations and LPSVD fits (left panels, top to bottom) of metMb (418 nm excitation), HRP/BHA (418 and $405 \mathrm{~nm}$ excitation), native HRP (418 and $405 \mathrm{~nm}$ excitation), and the H64L metMb mutant (405 nm excitation). The experiments were run in an open band detection scheme, and the respective frequency domain power spectra are shown in the right panels. The phases of the modes at 102 and $94 \mathrm{~cm}^{-1}$ in panels two and three are found to be $\sim \pi$, while the phases of the $46 \mathrm{~cm}^{-1}$ mode in H64LmetMb and the $39 \mathrm{~cm}^{-1}$ mode in metMb are $\sim \pi / 2$. The weaker mode near $37-39 \mathrm{~cm}^{-1}$ in HRP/BHA has a phase that is difficult to determine, but it appears to vary from $\sim-\pi / 2(418 \mathrm{~nm})$ to $\sim \pi / 4(405 \mathrm{~nm})$. 

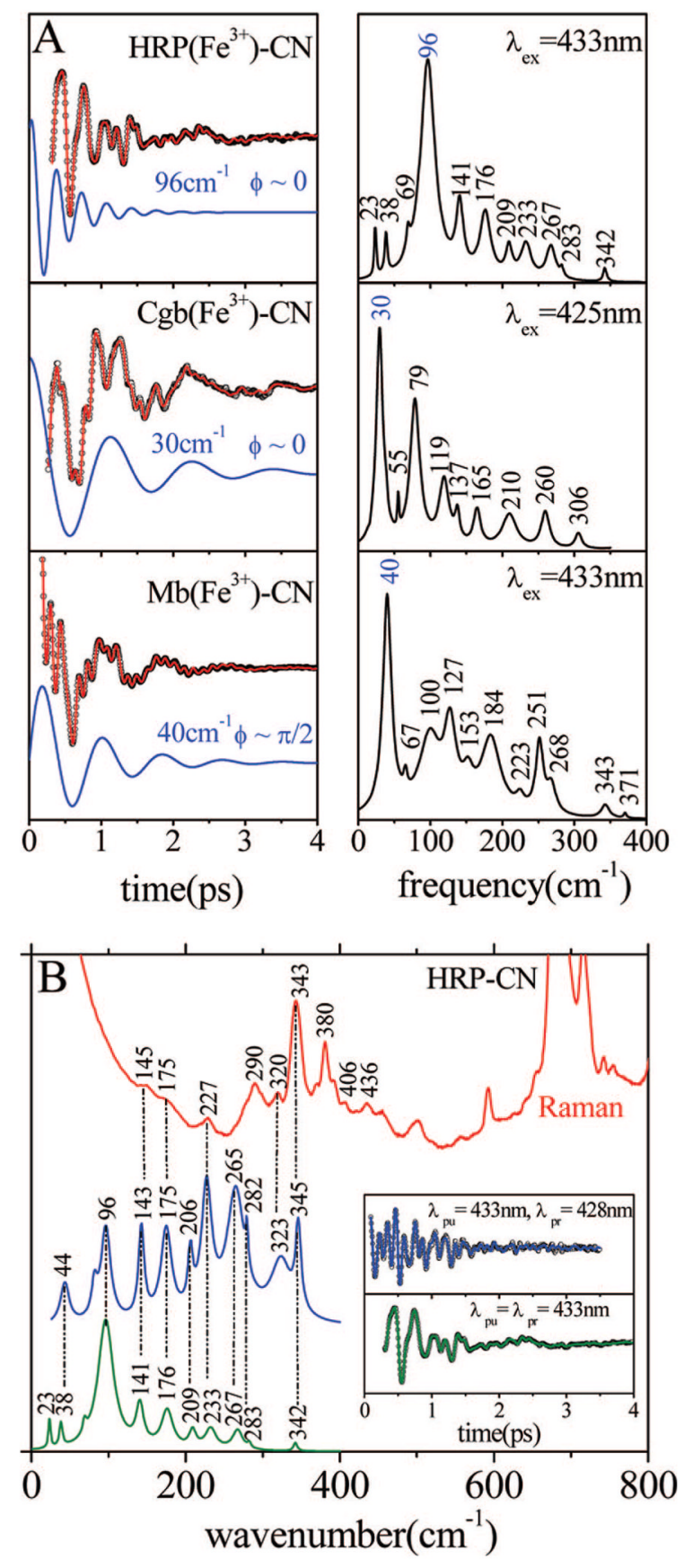

Figure 4.

(A) Coherent oscillations and the LPSVD fits (left panel) of the cyanide-bound complexes of $\mathrm{HRP}, \mathrm{Cgb}$, and $\mathrm{Mb}$. The respective power spectra are displayed in the right panels. The experiments were run in an open band detection scheme, with a $433 \mathrm{~nm}$ excitation wavelength for HRP and $\mathrm{Mb}$ and $425 \mathrm{~nm}$ for $\mathrm{Cgb}$. The dominant LPSVD components and their phases are shown displaced from the data. (B) Correlations between Raman and FCS spectra of the ferric HRP-CN sample. The inset shows the coherent oscillations and the LPSVD fits corresponding to open band (green) and dispersed (blue) detection. The Raman data were measured with a $413.1 \mathrm{~nm}$ excitation wavelength, while the FCS data were measured using a $433 \mathrm{~nm}$ excitation. 
The dispersed data were measured with a $0.5 \mathrm{~nm}$ spectral window, detuned $5 \mathrm{~nm}$ to the blue of the carrier frequency maximum $\left(\lambda_{\mathrm{pr}}=428 \mathrm{~nm}\right)$. 

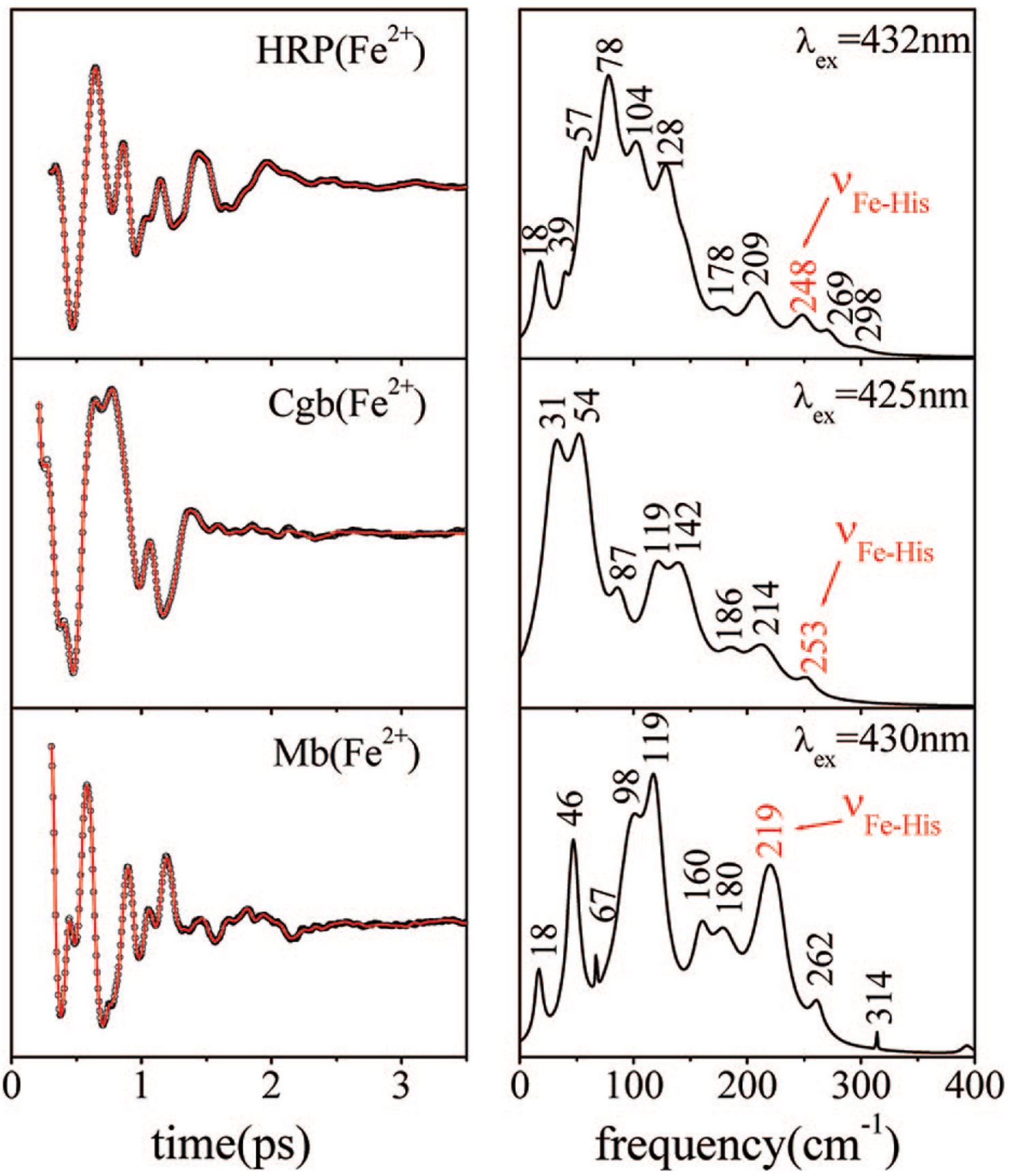

Figure 5.

Coherent oscillations and the LPSVD fits (left panel) of the reduced five-coordinate species of HRP, $\mathrm{Cgb}$, and $\mathrm{Mb}$. The respective power spectra are displayed in the right panels. The experiments were run in an open band detection scheme, with 432, 425, and $430 \mathrm{~nm}$ excitation wavelengths, respectively. The Fe-His stretching frequency near $219 \mathrm{~cm}^{-1}$ in deoxyMb is shifted up to $248 \mathrm{~cm}^{-1}$ in ferrous HRP and to $253 \mathrm{~cm}^{-1}$ in ferrous $\mathrm{Cgb}$. For Cgb, the phases of the modes at 31 and $54 \mathrm{~cm}^{-1}$ are near zero and $\pi / 2$, respectively. For $\mathrm{Mb}$, the phase of the mode at $46 \mathrm{~cm}^{-1}$ is $\sim 2 \pi / 3$, consistent with the phase and frequency "drifts" reported previously, 57 and associated with conformational inhomogeneity. 

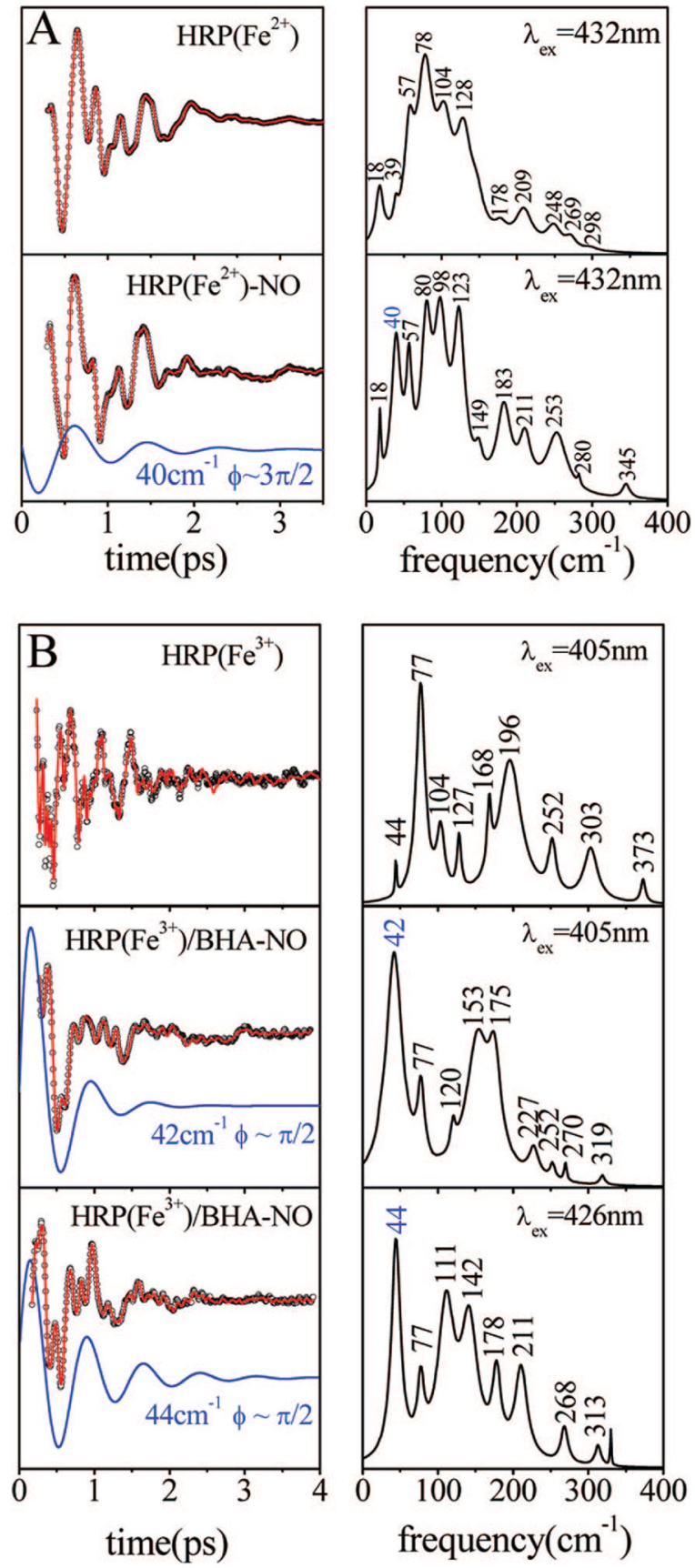

Figure 6.

(A) Coherent oscillations and the LPSVD fits (left panel) of reduced five-coordinate and reduced NO-bound species of HRP. The respective power spectra are displayed in the right panels. The mode at $40 \mathrm{~cm}^{-1}$ is activated upon NO photolysis and appears with a phase of $-\pi / 2$. The experiments were run in an open band detection scheme, with a $432 \mathrm{~nm}$ excitation wavelength. (B) Coherent oscillations and the LPSVD fits (left panel) of the native and ferric NO-bound species of HRP. The respective power spectra are displayed in the right panels. The experiments were run in an open band detection scheme. The excitation wavelength is shown for each sample. The dominant components at $42 \mathrm{~cm}^{-1}$ (405 nm excitation) and $44 \mathrm{~cm}^{-1}$ (426 $\mathrm{nm}$ excitation) and their phase are shown displaced from the data. 

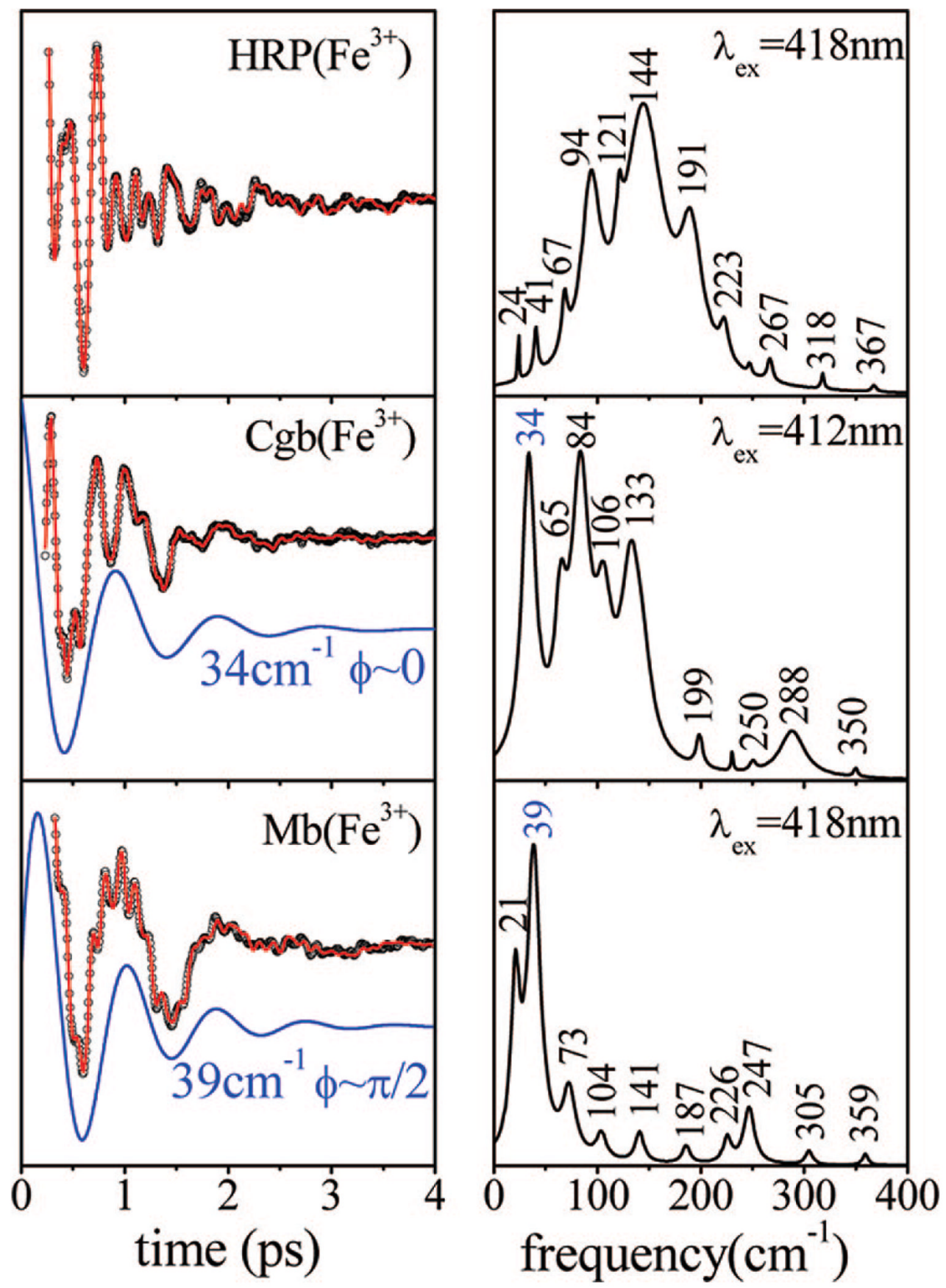

Figure 7.

Coherent oscillations and the LPSVD fits (left panel) of the native ferric species of HRP, Cgb, and $\mathrm{Mb}$. The respective power spectra are displayed in the right panels. The experiments were run in an open band detection scheme, with excitation at $418 \mathrm{~nm}$ for HRP and $\mathrm{Mb}$ and $412 \mathrm{~nm}$ for $\mathrm{Cgb}$. The $34 \mathrm{~cm}^{-1}$ mode of $\mathrm{Cgb}$ and the $39 \mathrm{~cm}^{-1}$ of $\mathrm{Mb}$ and their phases are shown displaced from the data. 

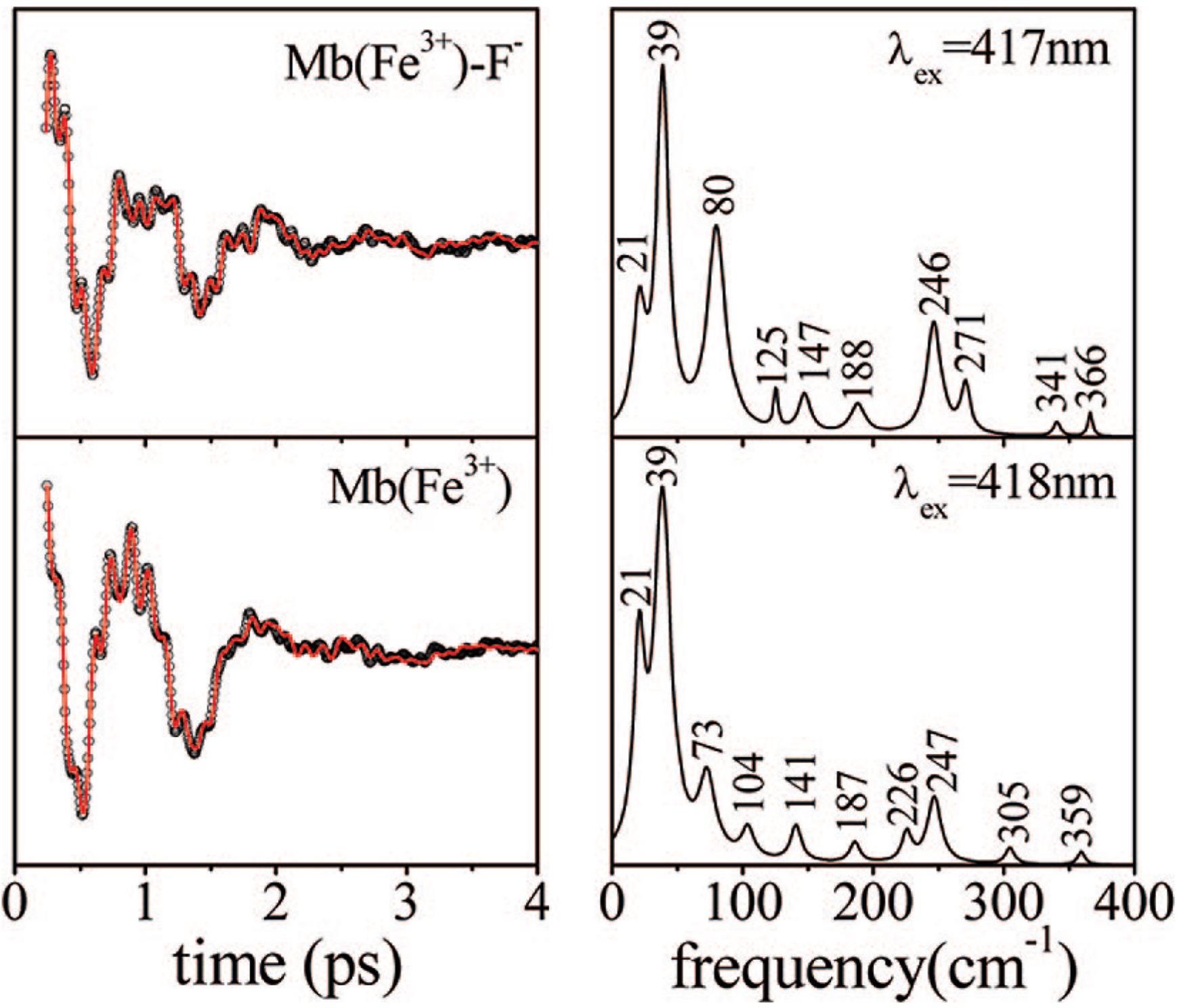

Figure 8.

Coherent oscillations and the LPSVD fits (left panel) of ferric $\mathrm{MbF}^{-}$and metMb. The respective power spectra are displayed in the right panels. The experiments were run in an open band detection scheme, with $417 \mathrm{~nm}$ and $418 \mathrm{~nm}$ excitation wavelengths, respectively. The phase of the strong modes at $39 \mathrm{~cm}^{-1}$ in both samples is $\pi / 2$. 

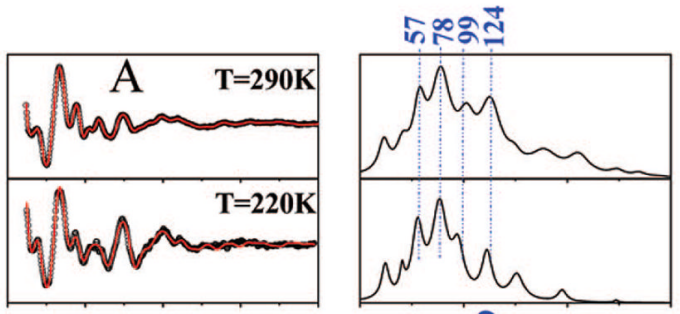

แัホลำ
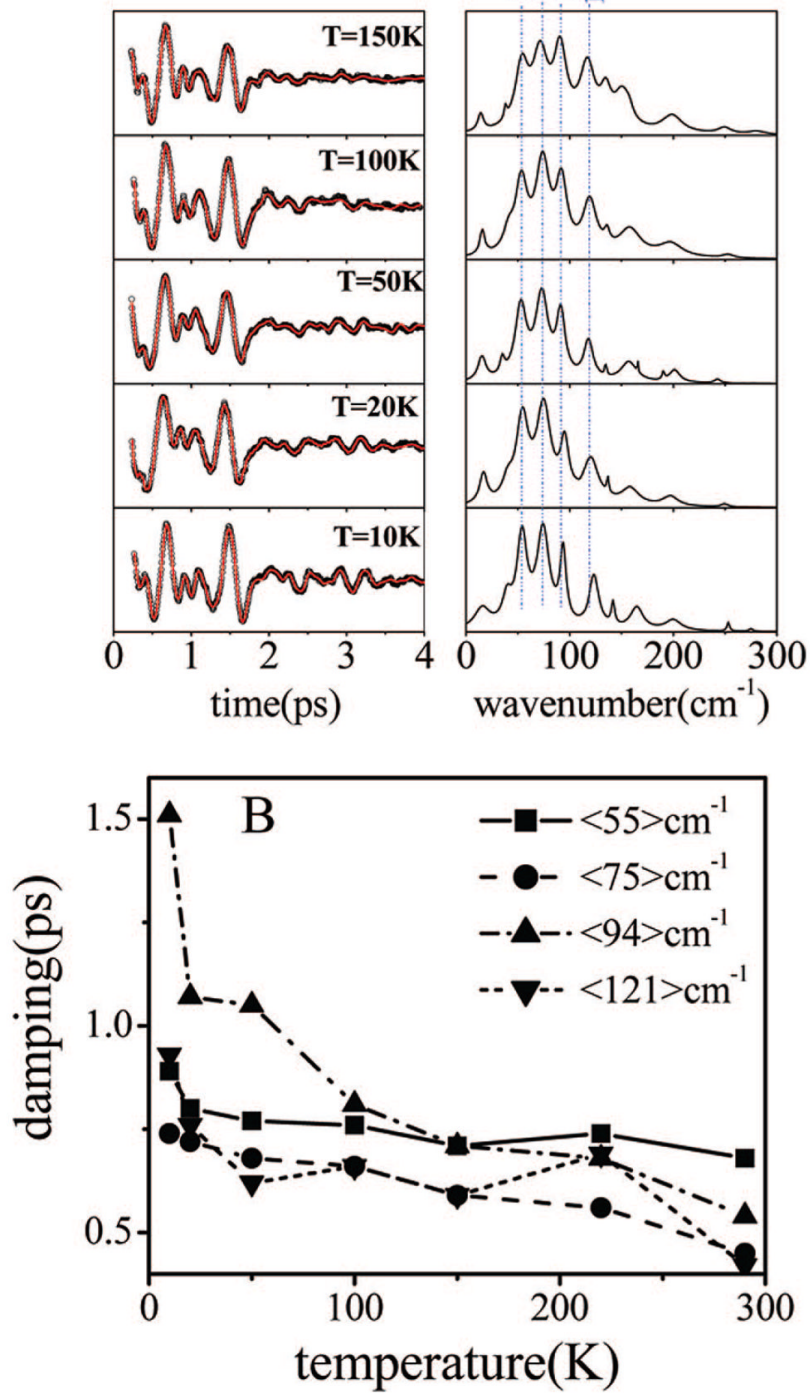

Figure 9.

(A) Temperature-dependent coherence spectra of ferrous HRP. Coherent oscillations and the LPSVD fits are shown in the left panels and the resulting power spectra are presented in the right panels. The samples were prepared in a mixture of $75 \%(\mathrm{v} / \mathrm{v})$ glycerol and phosphate buffer. The experiments were run in an open band detection scheme with a $432 \mathrm{~nm}$ excitation wavelength. The average mode frequencies above and below glass transition of the solvent are shown. (B) A plot of the damping times of the four principal components observed in the coherence spectra of ferrous HRP as a function of temperature. The modes are identified by their frequencies averaged over the entire temperature domain of the experiments. 

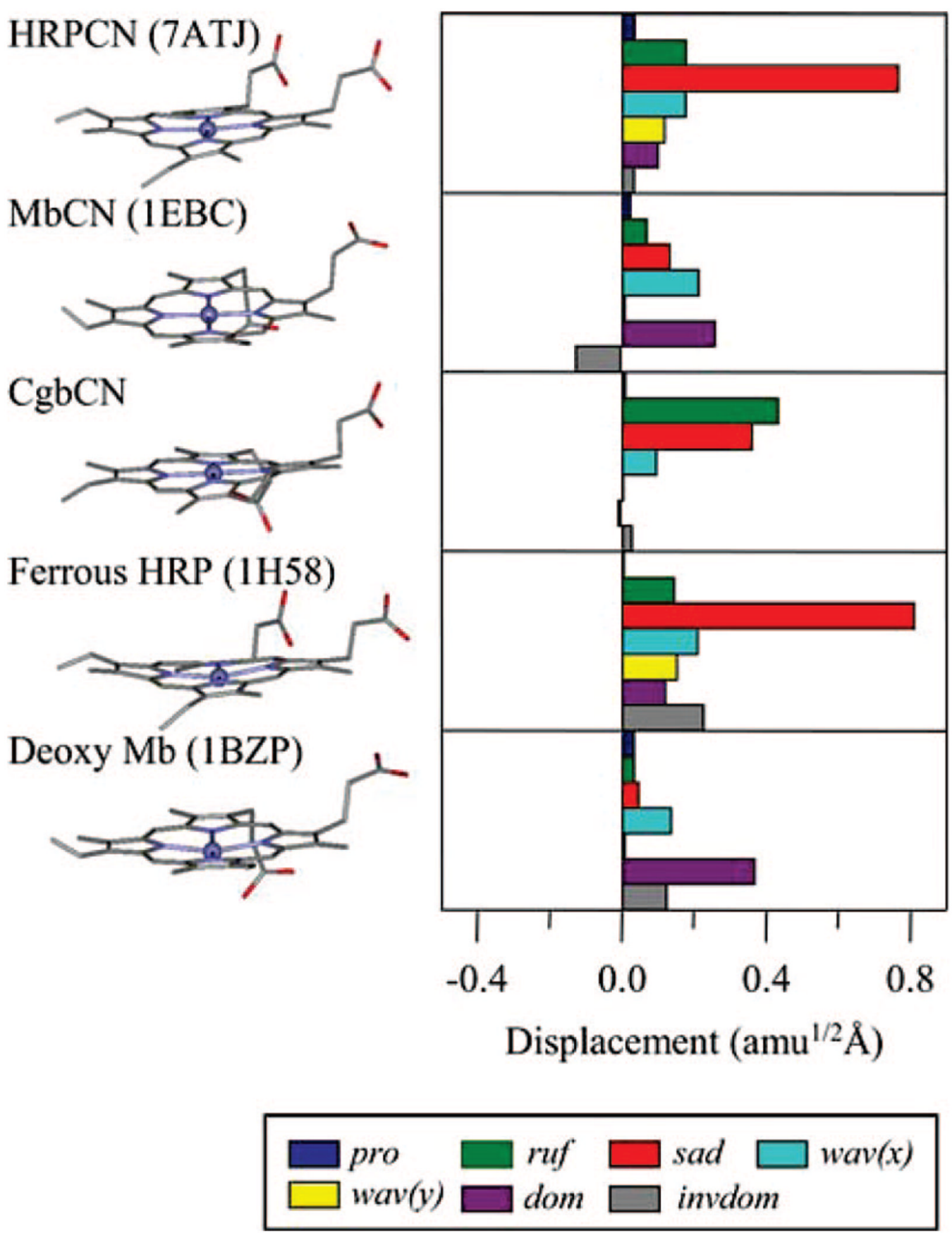

Figure 10.

Comparison of heme structures in HRP and Mb for the ferricyanide adducts and the ferrous forms. The heme in $\mathrm{Cgb}$ is shown for the ferricyanide adduct only. The NSD heme distortion analysis is in the right panel of the figure. A displacement of $1 \mathrm{amu}^{1 / 2} \AA$ represents that the square root of the sum of squares of the displacements of Fe and 24 porphyrin $(4 \mathrm{~N}, 20 \mathrm{C})$ atoms is $1 \mathrm{amu}^{1 / 2} \AA$. The color coding for the modes is pro, propellering (blue); ruf, ruffling (green); sad, saddling (red); wav(x), waving (light blue); wav(y), waving $_{\mathrm{y}}$ (yellow); dom, doming (purple); invdom, inverse doming (gray). The minus sign of displacement is defined only for doming and inverse doming to indicate the direction of Fe displacement (+, proximal; 
-, distal). The HRP and Mb structure files were downloaded from $\mathrm{PDB}$, and all structures were visualized with ViewerLite. The PDB ID numbers are shown in parenthesis. 


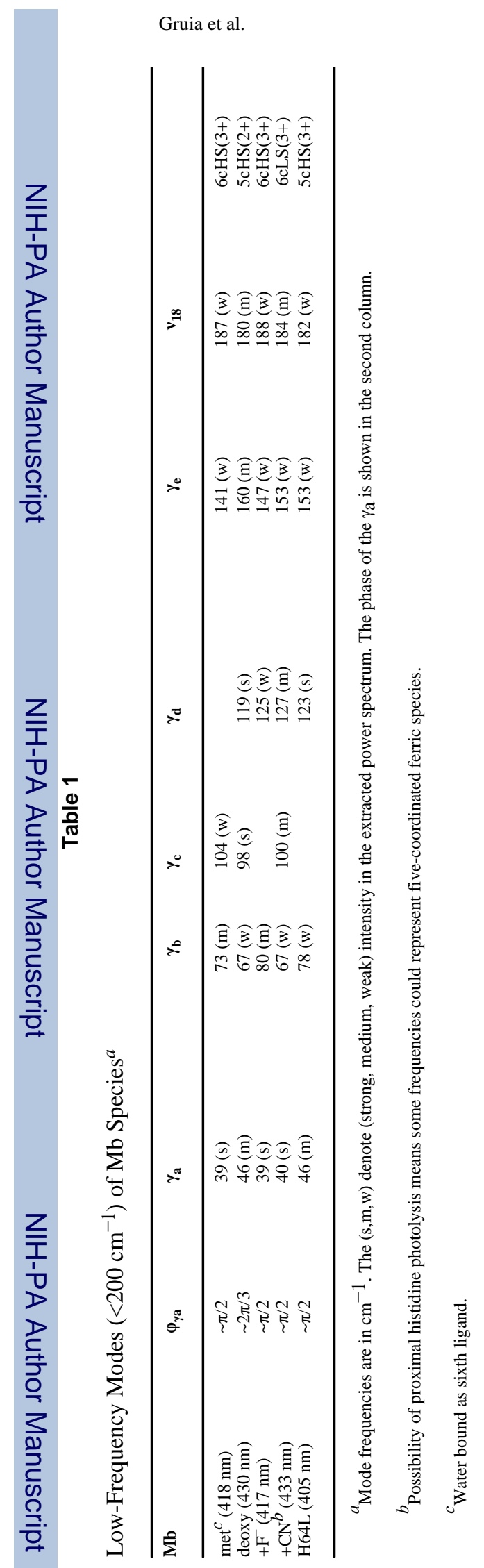

Page 32 


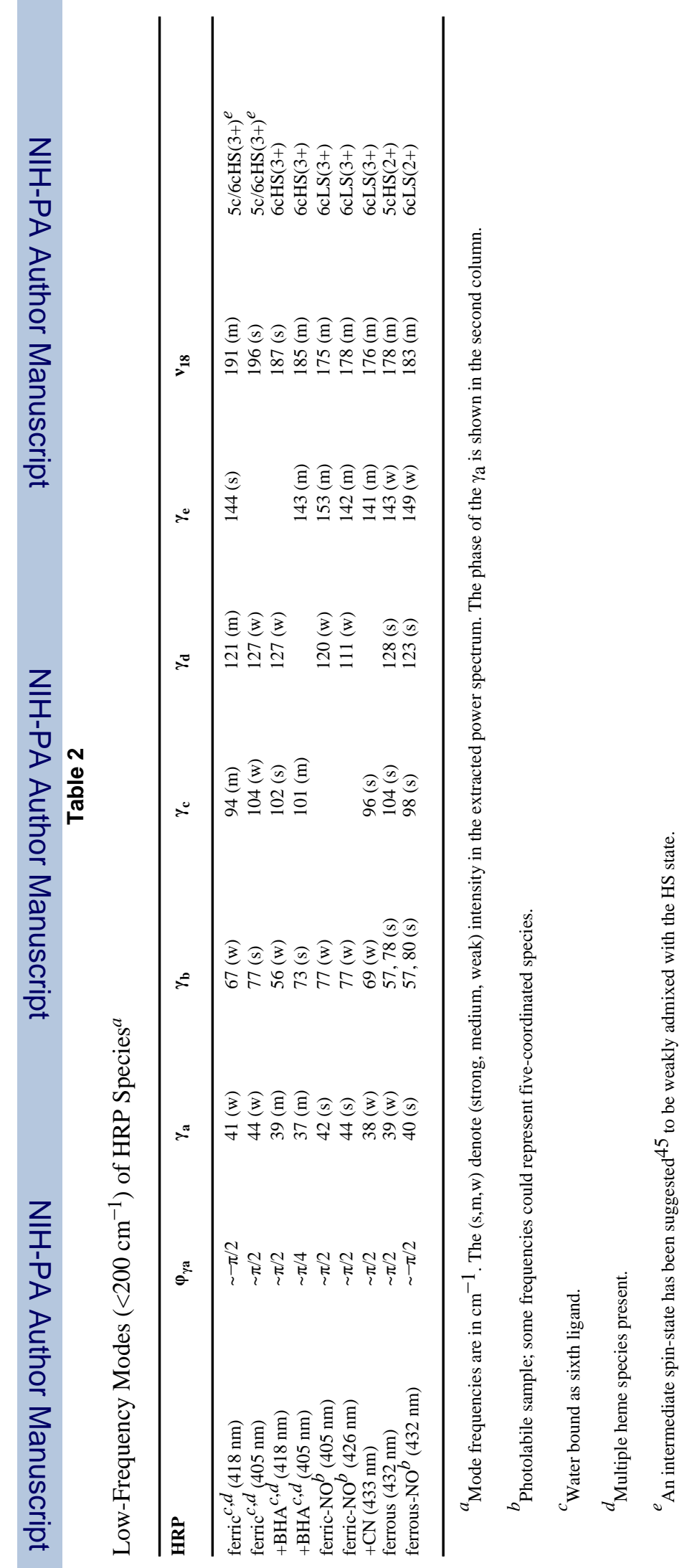




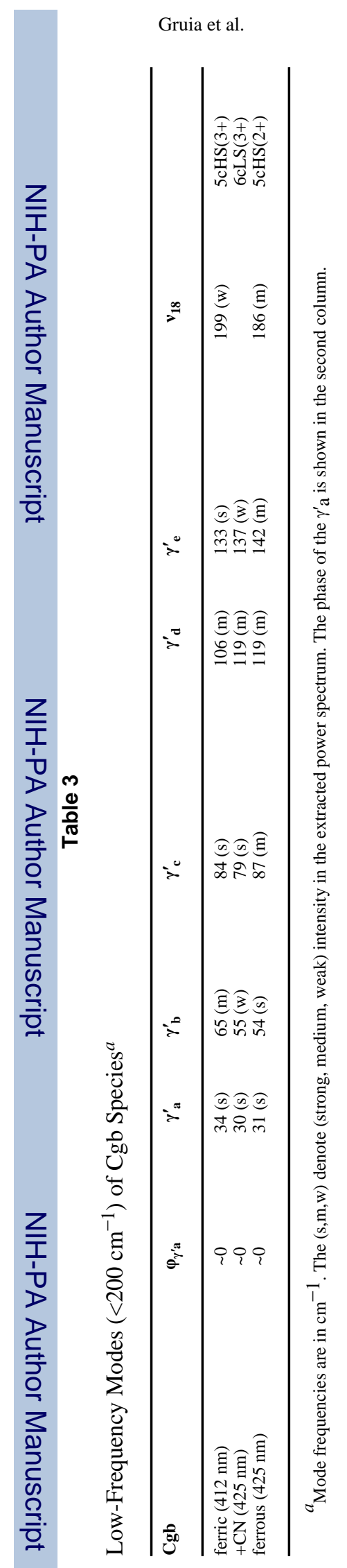

Page 34 
Table 4

Low-Frequency Modes of Ferrous HRP ${ }^{a}$

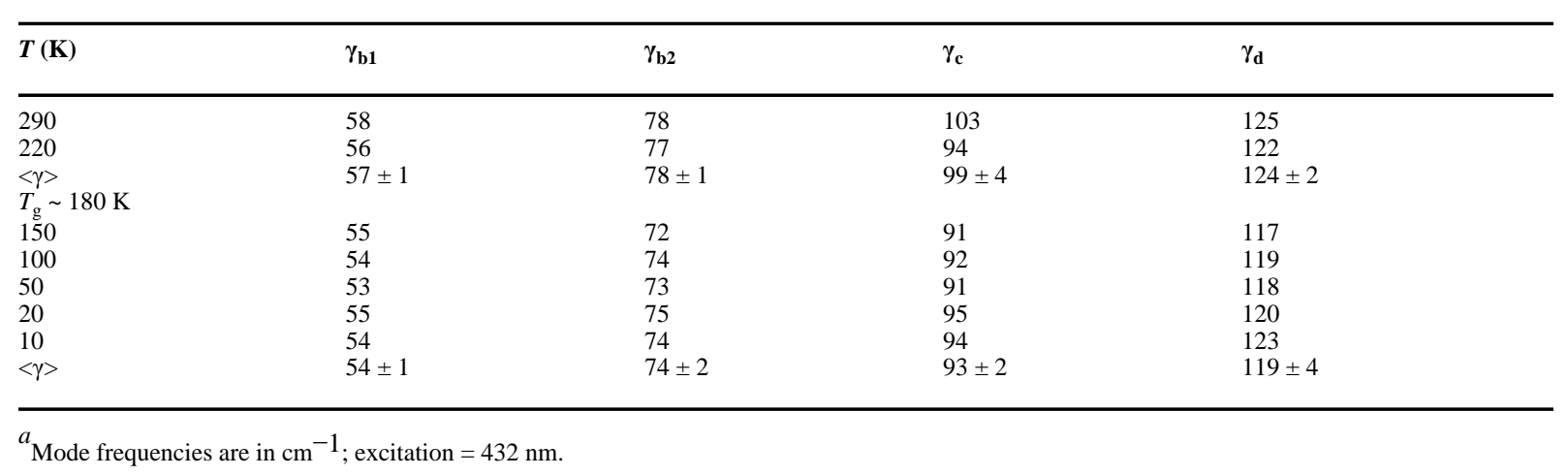

